\title{
Discovery and confirmation of the shortest gamma ray burst from a collapsar
}

Tomás Ahumada ${ }^{1,2,3}$, Leo P. Singer ${ }^{2,4}$, Shreya Anand ${ }^{5}$, Michael W. Coughlin ${ }^{6}$, Mansi M. Kasliwal ${ }^{5}$, Geoffrey Ryan ${ }^{1,2}$, Igor Andreoni ${ }^{5}$, S. Bradley Cenko ${ }^{3,4}$, Christoffer Fremling ${ }^{5}$, Harsh Kumar ${ }^{7,8}$, Peter T. H. Pang ${ }^{9,10}$, Eric Burns ${ }^{11}$, Virginia Cunningham ${ }^{1,2}$, Simone Dichiara ${ }^{1,2}$, Tim Dietrich ${ }^{12}$, Dmitry S. Svinkin ${ }^{13}$, Mouza Almualla ${ }^{14}$, Alberto J. Castro-Tirado ${ }^{15,16}$, Kishalay De ${ }^{5}$, Rachel Dunwoody $^{17}$, Pradip Gatkine ${ }^{5}$, Erica Hammerstein ${ }^{1}$, Shabnam Iyyani ${ }^{18}$, Joseph Mangan ${ }^{17}$, Dan Perley $^{19}$, Sonalika Purkayastha ${ }^{20}$, Eric Bellm ${ }^{21}$, Varun Bhalerao ${ }^{7}$, Bryce Bolin ${ }^{5}$, Mattia Bulla ${ }^{22}$, Christopher Cannella ${ }^{23}$, Poonam Chandra ${ }^{20,24}$, Dmitry A. Duev ${ }^{5}$, Dmitry Frederiks ${ }^{13}$, Avishay Gal-Yam ${ }^{25}$, Matthew Graham ${ }^{5}$, Anna Y. Q. Ho ${ }^{26,27}$, Kevin Hurley ${ }^{28}$, Viraj Karambelkar ${ }^{5}$, Erik C. Kool $^{29}$, S. R. Kulkarni ${ }^{5}$, Ashish Mahabal ${ }^{5}$, Frank Masci ${ }^{30}$, Sheila McBreen ${ }^{17}$, Shashi B. Pandey ${ }^{31}$, Simeon Reusch ${ }^{32,33}$, Anna Ridnaia ${ }^{13}$, Philippe Rosnet ${ }^{34}$, Benjamin Rusholme ${ }^{30}$, Ana Sagués Carracedo ${ }^{35}$, Roger Smith ${ }^{36}$, Maayane Soumagnac ${ }^{25,37}$, Robert Stein ${ }^{32,33}$, Eleonora Troja ${ }^{3,1}$, Anastasia Tsvetkova ${ }^{13}$, Richard Walters ${ }^{36}$, and Azamat F. Valeev ${ }^{38}$

${ }^{1}$ Department of Astronomy, University of Maryland, College Park, MD 20742, USA

${ }^{2}$ Astrophysics Science Division, NASA Goddard Space Flight Center, MC 661, Greenbelt, MD 20771, USA

${ }^{3}$ Center for Research and Exploration in Space Science and Technology, NASA Goddard Space Flight Center, Greenbelt, MD 20771, USA

${ }^{4}$ Joint Space-Science Institute, University of Maryland, College Park, MD 20742, USA

${ }^{5}$ Division of Physics, Mathematics, and Astronomy, California Institute of Technology, Pasadena, CA 91125, USA

${ }^{6}$ School of Physics and Astronomy, University of Minnesota, Minneapolis, Minnesota 55455, USA

${ }^{7}$ Indian Institute of Technology Bombay, Powai, Mumbai 400076, India

${ }^{8}$ LSSTC Data Science Fellow

${ }^{9}$ Nikhef, Science Park 105, 1098 XG Amsterdam, The Netherlands

${ }^{10}$ Department of Physics, Utrecht University, Princetonplein 1, 3584 CC Utrecht, The Netherlands

${ }^{11}$ Louisiana State University, Baton Rouge, LA 70803, USA

${ }^{12}$ Institut für Physik und Astronomie, Universität Potsdam, Haus 28, Karl-Liebknecht-Str. 24/25, 14476, Potsdam, Germany

${ }^{13}$ Ioffe Institute, Polytekhnicheskaya, 26, St. Petersburg, 194021 - Russian Federation 
${ }^{14}$ American University of Sharjah, Physics Department, PO Box 26666, Sharjah, UAE

${ }^{15}$ Instituto de Astrofísica de Andalucía (IAA-CSIC), Glorieta de la Astronomía s/n, E-18008, Granada, Spain

${ }^{16}$ Departamento de Ingeniería de Sistemas y Automática, Escuela de Ingenieros Industriales, Universidad de Málaga, Unidad Asociada al CSIC, C. Dr. Ortiz Ramos sn, 29071 Málaga, Spain

${ }^{17}$ School of Physics, University College Dublin, Dublin 4, Ireland

${ }^{18}$ Inter-University Centre for Astronomy and Astrophysics, Pune, 411007, India

${ }^{19}$ Astrophysics Research Institute, Liverpool John Moores University, IC2, Liverpool Science Park, 146 Brownlow Hill, Liverpool L3 5RF, UK

${ }^{20}$ National Centre for Radio Astrophysics, Tata Institute of Fundamental Research, Pune University Campus, Ganeshkhind, Pune 411 007, India

${ }^{21}$ DIRAC Institute, Department of Physics and Astronomy, University of Washington, 3910 15th Avenue NE, Seattle, WA 98195, USA

${ }^{22}$ Nordita, KTH Royal Institute of Technology and Stockholm University, Roslagstullsbacken 23, 10691 Stockholm, Sweden

${ }^{23}$ Duke University, Electrical and Computer Engineering, Durham, NC 27708, USA

${ }^{24}$ Swarna Jayanti Fellow, Department of Science \& Technology, India

${ }^{25}$ Department of Particle Physics and Astrophysics, Weizmann Institute of Science, Rehovot, 76100 , Israel

${ }^{26}$ Miller Institute for Basic Research in Science, University of California, Berkeley, CA 94720, USA

${ }^{27}$ Department of Astronomy, University of California - Berkeley, Berkeley, CA 94720-5800, USA

${ }^{28}$ Space Sciences Laboratory, University of California - Berkeley, Berkeley, CA 94720-7450, USA

${ }^{29}$ The Oskar Klein Centre, Department of Astronomy, Stockholm University, AlbaNova, SE-106 91 Stockholm, Sweden

${ }^{30}$ IPAC, California Institute of Technology, 1200 E. California Blvd, Pasadena, CA 91125, USA

${ }^{31}$ Aryabhatta Research Institute of Observational Sciences (ARIES), Nainital, Uttrakhand, India

${ }^{32}$ Deutsches Elektronen Synchrotron DESY, Platanenallee 6, 15738 Zeuthen, Germany

${ }^{33}$ Institut für Physik, Humboldt-Universität zu Berlin, D-12489 Berlin, Germany

${ }^{34}$ Université Clermont Auvergne, CNRS/IN2P3, Laboratoire de Physique de Clermont, F-63000 Clermont-Ferrand, France

${ }^{35}$ The Oskar Klein Centre, Department of Physics, Stockholm University, AlbaNova, SE-106 91 Stockholm, Sweden 
${ }^{36}$ Caltech Optical Observatories, California Institute of Technology, Pasadena, CA 91125, USA

${ }^{37}$ Lawrence Berkeley National Laboratory, 1 Cyclotron Road, Berkeley, CA 94720, USA

${ }^{38}$ Special Astrophysical Observatory, Russian Academy of Sciences, Nizhnii Arkhyz, 369167 Russia

Gamma-ray bursts (GRBs) are among the brightest and most energetic events in the universe. The duration and hardness distribution of GRBs has two clusters ${ }^{1}$, now understood to reflect (at least) two different progenitors ${ }^{2}$. Short-hard GRBs (SGRBs; $T_{90}<2 \mathrm{~s}$ ) arise from compact binary mergers, while long-soft GRBs (LGRBs; $T_{90}>2 \mathrm{~s}$ ) have been attributed to the collapse of peculiar massive stars (collapsars) ${ }^{3}$. The discovery of SN 1998bw/GRB 980425 $5^{4}$ marked the first association of a LGRB with a collapsar and AT 2017gfo $/$ /GRB 170817A/GW170817 marked the first association of a SGRB with a binary neutron star merger, producing also gravitational wave (GW). Here, we present the discovery of ZTF20abwysqy (AT2020scz), a fast-fading optical transient in the Fermi Satellite and the InterPlanetary Network (IPN) localization regions of GRB 200826A; $X$-ray and radio emission further confirm that this is the afterglow. Follow-up imaging (at rest-frame 16.5 days) reveals excess emission above the afterglow that cannot be explained as an underlying kilonova $(\mathrm{KN})$, but is consistent with being the supernova (SN). Despite the GRB duration being short (rest-frame $T_{90}$ of $0.65 \mathrm{~s}$ ), our panchromatic follow-up data confirms a collapsar origin. GRB 200826A is the shortest LGRB found with an associated collapsar; it appears to sit on the brink between a successful and a failed collapsar. Our discovery is consistent with the hypothesis that most collapsars fail to produce ultra-relativistic jets.

On August 26, 2020, at 04:29:52 UT, the Gamma-ray Burst Monitor (GBM) on board the Fermi Gamma-ray Space Telescope detected GRB 200826A with duration $\left(T_{90}\right)$ of $1.14 \pm$ 0.13 seconds in the 50-300 keV energy rangeIn addition to Fermi (GBM trigger 620108997), GRB 200826A was detected by four other Interplanetary Network (IPN) instruments (see Methods).

The gamma-ray properties alone do not always yield an unambiguous classification. Some SGRBs show afterglow and host properties akin to LGRBs, e.g. Ref. 7; some LGRBs show no evidence for collapsars to deep limits akin to SGRBs, e.g. Ref. ${ }^{8}$. Based solely on $T_{90}{ }^{9}$, GRB 200826A has a SGRB probability of $65 \%_{-11}^{+12}$. Also taking into consideration the $E_{\text {peak }}$ parameter of a Comptonized model fit to the single spectrum over the duration of the burst (see 
Extended Data Table 5), the probability that GRB 200826A is a SGRB increases to 74\% (see Figure 1 and Methods). However, based on rest-frame energetics, GRB 200826A is not consistent with the SGRB population (right panel in Extended Data Figure 1).

Starting 4.2 hours after the GRB, we observed 180 sq. degrees of the Fermi-GBM localization with the Zwicky Transient Facility (ZTF) ${ }^{10}$ (see Fig. 2). At 17 hours after the GRB, IPN triangulated the source to a smaller region. ZTF20abwysqy was the only candidate that passed our alert filtering scheme and was also inside the IPN region (see Methods). We discovered ZTF20abwysqy at a brightness of $g=20.86 \pm 0.04 \mathrm{mag}$ (AB system). The previous upper limit $(g>21.3 \mathrm{mag}$ at $5 \sigma$ ) was 17.3 hours before the GRB, as part of the nominal all-sky survey mode. In addition to the spatial coincidence, ZTF20abwysqy was associated with a fading X-ray counterpart ${ }^{11}$ and variable radio emission ${ }^{12}$, confirming ZTF20abwysqy as the afterglow of GRB 200826A (see Extended Data Table 1|Extended Data Table 3 and Extended Data Table 4).

ZTF20abwysqy was discovered in a galaxy with archival detections in Pan-STARRS 1 (PS1) and Legacy Survey $(\mathrm{LS})^{14}$, at a LS photometric redshift of $z_{p}=0.71 \pm 0.14$. The offset between the host galaxy's centroid and the transient is $0.18 \pm 0.05^{\prime \prime}$, corresponding to a physical distance of $2.09 \pm 0.6 \mathrm{kpc}$. We acquired a Gran Telescopio Canarias (GTC) spectrum of the galaxy; we see strong [OII] and [OIII] lines at $z=0.748$ (see Extended Data Table 2 and Extended Data Figure 2). With both spectral and photometric spectral energy distribution (SED) fitting, we infer a stellar mass for the host galaxy of $\sim 10^{9.7} M_{\odot}$ (see Extended Data Figure 2, Extended Data Figure 3 and Extended Data Table 6 in Methods), which is near the distribution peak for LGRBs ${ }^{15}$, while below the median for SGRBs based on Ref. 16.

We model the GRB afterglow using the standard synchrotron fireball model to constrain parameters related to the energy and geometry of the GRB central engine (see Methods). Electrons in the circumburst medium are accelerated by the shock wave and reach a power-law energy distribution characterized by the index $p, N(E) \propto E^{-p}$. This results in a SED described by a series of broken power laws, e.g. Ref. ${ }^{17}$ (See Extended Data Figure 4 and Extended Data Figure 5). The associated isotropic kinetic energy of $E_{\mathrm{K} \text {,iso }}=6.0_{-4.4}^{+51.3} \times 10^{52} \mathrm{erg}$ lies in the top $5 \%$ of the $E_{K, \text { iso }}$ distribution for SGRBs ${ }^{16}$, but within the $90 \%$ confidence range of the LGRB energy distribution ${ }^{18}$ (see Methods). Our data can only loosely constrain the circumburst density. The upper end of the distribution is consistent with the values found for the median circumburst densities ${ }^{19}$ of LGRBs, 
while the lower end is more representative of $\mathrm{SGRB} s{ }^{16}$.

In the collapsar scenario, a high-velocity stripped-envelope SN (SN Ic-BL) should follow the GRB detection ${ }^{3}$. To test this scenario, we used the Gemini Multi-Object Spectrograph (GMOS-N; see Methods) to acquire $r$ - and $i$-band images of ZTF20abwysqy on three different epochs: $\sim 28$, $\sim 45$, and $\sim 80$ days after the GRB trigger (epoch 1, 2 and 3 respectively). Using epoch 3 as the reference, we undertook image subtraction using two different subtraction algorithms (see Methods for details). On epoch 1, our Gemini observations show evidence of a transient with an $i$-band magnitude of $25.45 \pm 0.15 \mathrm{mag}$ (with an $i$-band $5 \sigma$ limit of $25.9 \mathrm{mag}$, see Extended Data Table 1); there is no source in the $r$-band observations up to a $5 \sigma$ limit of 25.4 mag (see Fig. 3 ). On epoch 2, we do not detect a source up to a $5 \sigma$ limit of 25.5 mag in the $i$-band and 25.7 mag in the $r$-band. Thus, at a rest-frame time of $\sim 16$ days, the foreground extinction-corrected absolute magnitude of ZTF20abwysqy is $M_{i}=-18.0 \mathrm{mag}$.

To understand the source of the $i$-band excess, we use Markov chain Monte Carlo (MCMC) and full forward modeling of all multi-band observations excluding the GMOS detection to compare three scenarios: an afterglow only, an afterglow plus a KN, and an afterglow plus a SN. The $\mathrm{KN}$ model is based on a best-fit template to AT2017 $\mathrm{gfo}^{20}$ scaled by a compilation of SGRB-KN candidates $^{21}$; the collapsar model uses a SN1998bw template ${ }^{22}$ with stretch and scale parameters drawn from a prior that is consistent with the historical GRB-SN sample ${ }^{23}$. We dismiss the afterglow-only and afterglow-plus-KN models because they predict $i$-band flux at the time of the GMOS data point that is too faint by $1.6_{-0.3}^{+1.8} \mathrm{mag}$ - inconsistent with the observations at the $\sim 5 \sigma$ level (see Extended Data Figure 6 and Extended Data Table 7).

We repeated the analysis of all three scenarios including the GMOS data point in order to do Bayesian model comparison. The Bayes factor between the afterglow-plus-KN and afterglow-only models is $\sim 1$, indicating that neither model is favored over the other. However, the Bayes factor between the afterglow-plus-SN and afterglow-only model is $10^{5.5}$, indicating that the afterglow-plus-SN model is strongly favored. Based on the compilation of Ref. ${ }^{23}$ of GRBs with associated SNe, GRB 200826A is the shortest LGRB found with an associated collapsar, with an observed $T_{90}$ of $1.13 \mathrm{~s}$ and rest-frame $T_{90}$ of $0.65 \mathrm{~s}$ (see Figure 1). The second shortest LGRB with a SN is GRB 040924, with a rest-frame $T_{90}$ of $1.29 \mathrm{~s}^{23}$. 
In the conventional fireball model of a LGRB, the rest-frame duration of the prompt emission, $t_{\gamma}$, is the difference between the duration of the activity of the central engine, $t_{e}$, and the time required to break out of the envelope, $t_{b}{ }^{24}$. The short duration of GRB 200826A suggests that $t_{e} \gtrsim$ $t_{b}$, which might imply that its central engine is active only very briefly, or that the stellar envelope is unusually thick, as compared to other LGRBs, perhaps requiring a nonstandard progenitor ${ }^{25}$. Then it is natural to infer that there must be collapsars for which $t_{e}<t_{b}$ that fail to produce a fireball. For $t_{e} \lesssim t_{b}$, the jet may fail to clear a path for itself and remains cocooned within the exploding star, yet the cocoon itself may still produce a mildly relativistic shock breakout that manifests as a soft, quasi-thermal, low-luminosity GRB (llGRB) like GRB 980425/SN 1998bw or GRB 060218/SN2006aj26/27. For $t_{e} \ll t_{b}$, there may be no prompt emission at all.

GRB 200826A, then, may sit on the brink between a successful LGRB and a failed one. The sign of one continuous physical parameter, $t_{e}-t_{b}$, switches a collapsar-powered GRB discontinuously between two dominant emission mechanisms: internal shocks in a relativistic jet or mildly relativistic shock breakout at the surface of the exploding star. These two mechanisms correspond to two widely separated and disconnected regions in the hardness-duration-fluence phase space: the traditional border between SGRBs and LGRBs, and the exceptionally long and soft region occupied by llGRBs and X-ray flashes (XRFs). The local rate of LGRBs $\left(\sim 1 \mathrm{Gpc}^{-3} \mathrm{yr}^{-1}\right)^{27}$ is 2 orders of magnitude lower than the rate of llGRBs $\left(300 \mathrm{Gpc}^{-3} \mathrm{yr}^{-1}\right)^{27}$ and 3 orders of magnitude lower than the rate of SNe Ic-BL $\left(4500 \mathrm{Gpc}^{-3} \mathrm{yr}^{-1}\right)^{28}$. It is understood that LGRBs, and to a lesser extent, llGRBs, are collimated and relativistically beamed, suppressing the detection of events in which the jet is pointed away from our line of sight. However, even allowing for a beaming correction to their rates of 100 for LGRBs and 1-10 for 11 GRBs ${ }^{27}$ (but no beaming correction for SNe), LGRBs still occur at a rate $\left(100 \mathrm{Gpc}^{-3} \mathrm{yr}^{-1}\right)$ that is up to an order of magnitude lower than that of llGRBs (300-3000 $\mathrm{Gpc}^{-3} \mathrm{yr}^{-1}$ ) or that of SNe Ic-BL, suggesting that majority of the collapsars fail to produce an ultra-relativistic jet and instead drive a wide-angle or nearly isotropic and only mildly-relativistic cocoon.

Based on indirect evidence from the host, afterglow, and gamma-ray properties, it has been argued that as many as $84 \%$ of bursts detected by the Neil Gehrels Swift Observatory ${ }^{29}$ (Swift) and $40 \%$ of the Fermi bursts that are nominally SGRBs $\left(T_{90}<2 \mathrm{~s}\right)$ are actually misclassified LGRBs ${ }^{30}$. If this is correct, then one would expect many more short-duration GRBs with collapsars that are on the edge between success and failure of the jet. The discovery of GRB 200826A, an SGRB 
imposter, lends credence to this bold claim, and would suggest that the rates of such short-duration LGRBs is comparable to the rate of llGRBs, up to a few hundred $\mathrm{Gpc}^{-3} \mathrm{yr}^{-1}$. Thus, our discovery upholds the hypothesis that most collapsars fail to produce jets. 


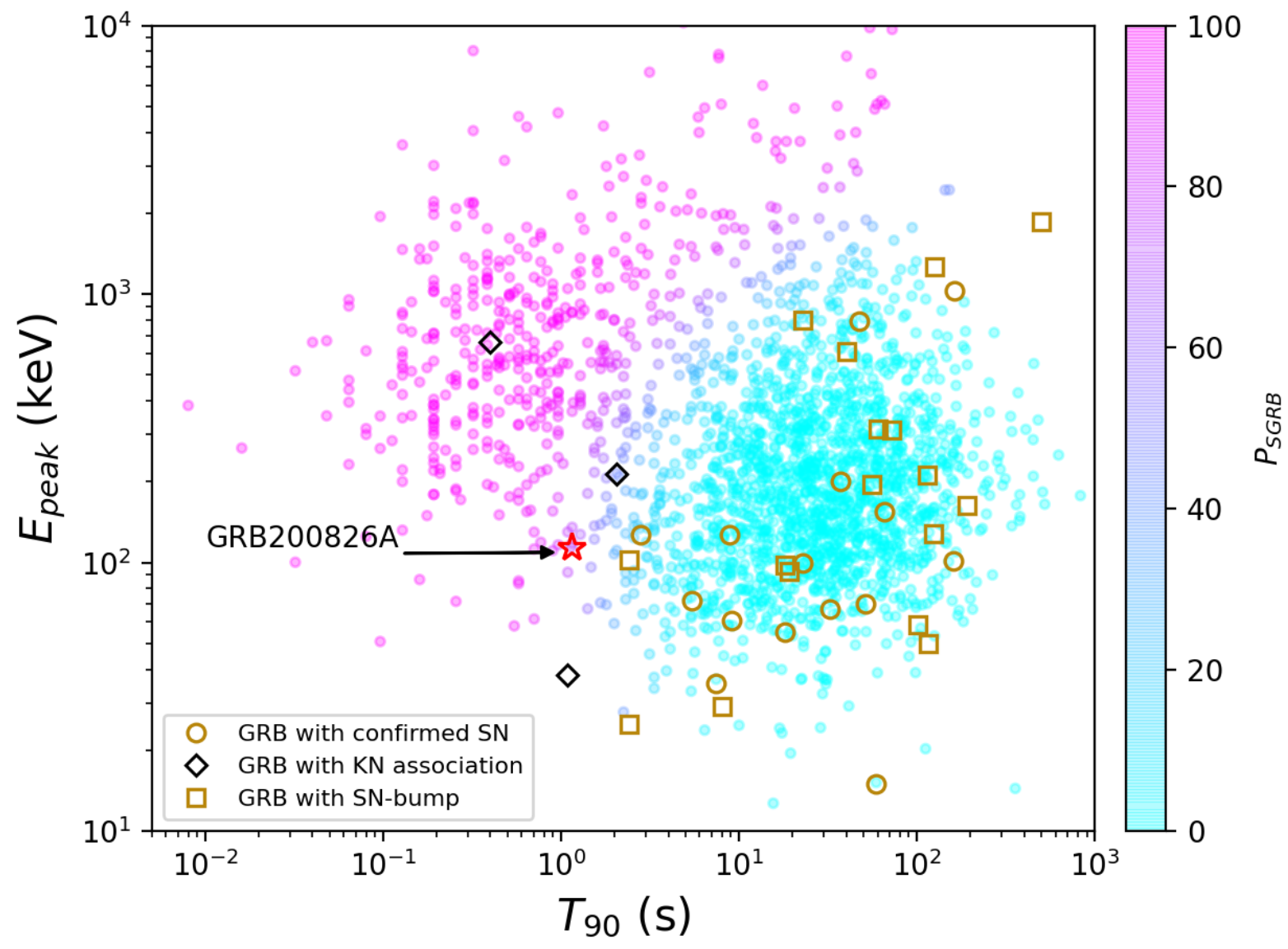

Figure 1: Gamma-ray properties of GRB 200826A in context. The peak energy based on a Comptonised fit, $E_{\text {peak }}(\mathrm{keV})$, vs. the time-integrated, $\mathrm{T}_{90}$ (s), for 2310 Fermi GBM GRBs. The data are fit with two log-normal distributions for the two GRB classes. The color of the data points indicate the probability with magenta being $100 \%$ SGRB and cyan being 100\% LGRB. GRB 200826A is surrounded by a red star with a SGRB probability of $74 \%$ (See Methods). Yellow squares show LGRBs with SN-bumps, yellow circles show LGRBs with spectroscopically confirmed $\mathrm{SN}^{23}$, black diamonds show $\mathrm{SGRB}$ s with claimed $\mathrm{KN}$ excess ${ }^{21}$. 


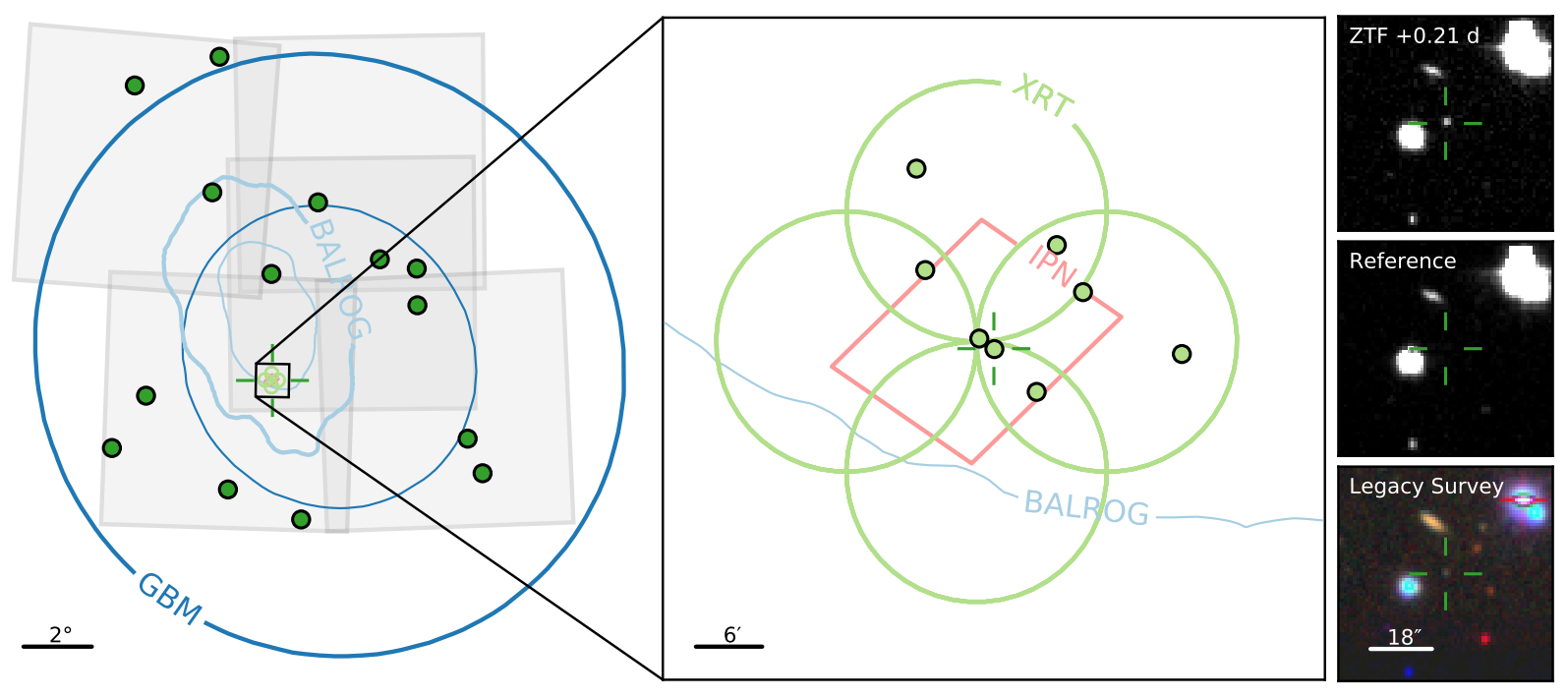

Figure 2: Discovery of the afterglow of GRB 200826A. It was found at the position $\alpha=$ $00^{h} 27^{m} 08.542^{s}, \delta=+34^{h} 01^{m} 38.327^{s}$ (J2000) with an uncertainty of 0.08 ." Contours in the left panel represent the Fermi GBM 90\% (thick) and 50\% (thin) credible regions from the official Fermi GBM localization (dark blue) and BALROG (light blue). The filled gray squares show the ZTF fields observed and the dark green dots are the positions of the ZTF Night 1 optical candidates. In the middle panel, $3 \sigma$ IPN triangulation is shown in pink; the four fields of the four XRT tiled observations are shown as light green circles and the XRT candidates are light green dots. The position of the afterglow is marked by the dark green reticle. The right-hand panels are centered at the position of ZTF20abwysqy. The cutouts, from top to bottom, show the ZTF discovery image, the ZTF stacked reference image, and a false color image showing the host galaxy from Legacy Survey DR8. In the cutouts, North is up and East to the left. 


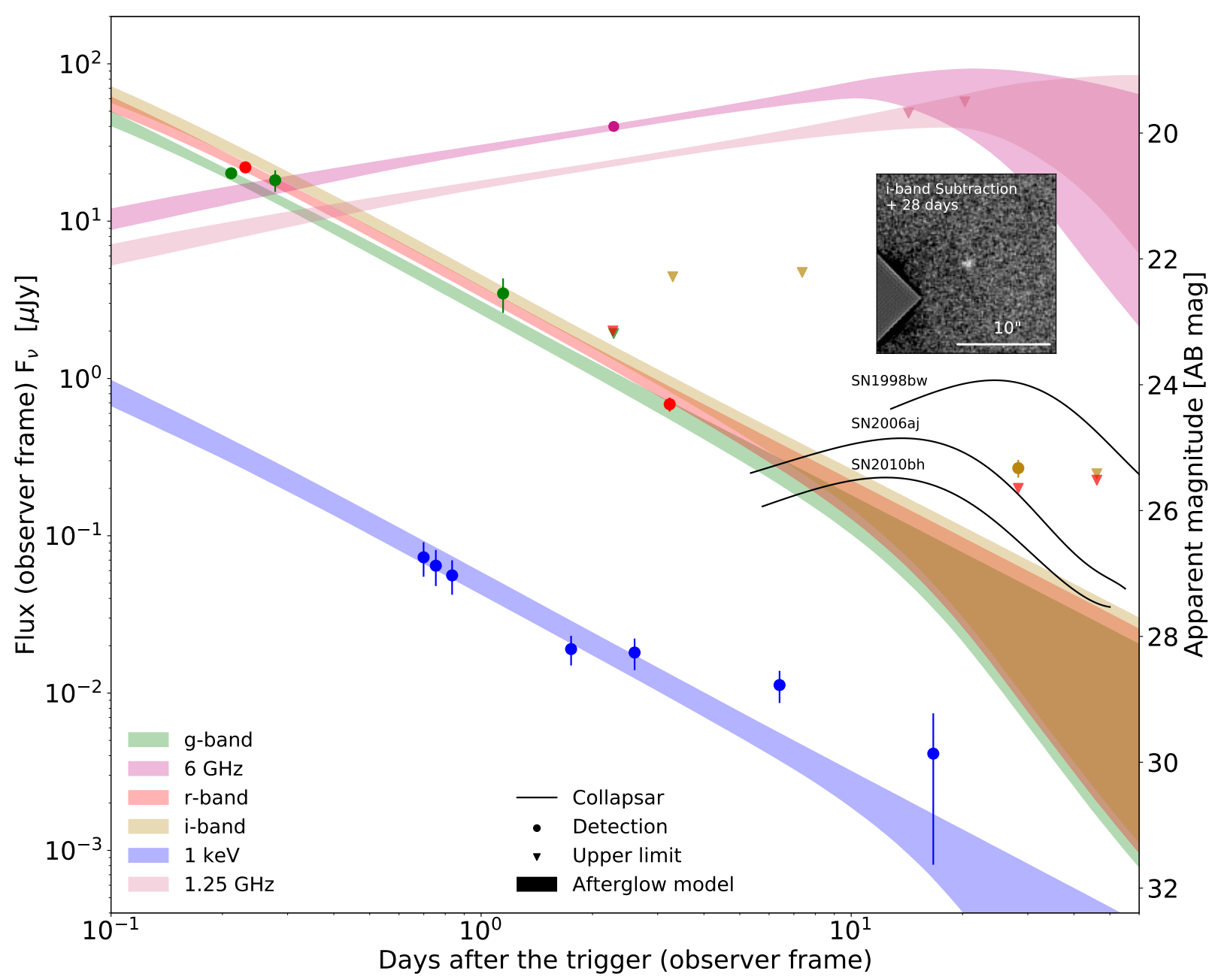

Figure 3: Panchromatic afterglow and collapsar confirmation. The available multiwavelength light curve data is over-plotted with the best fits from the afterglowpy modeling assuming an ISM-like environment. Detections are shown as circles with their respective error bars, and upper limits are shown as inverted triangles. The optical $g$-, $r$ - and $i$ - bands are shown in green, red and yellow, the XRT $1 \mathrm{keV}$ data is shown in blue, the VLA in fuchsia, while the GMRT data is presented in pink. We show the K-corrected light curves of three well-studied GRB-SNe (SN1998bw, one of the brightest; SN2006aj and SN2010bh, two of the faintest) with solid black lines. The Gemini GMOS-N $i$-band detection is shown at day 28.28, as a yellow circle, and is consistent with the collapsar population. We show a cutout of the $i$-band ZOGY subtraction, revealing our $25.45 \mathrm{mag}$ detection with a ZOGY corrected score of 4. 
1. Kouveliotou, C. et al. Identification of two classes of gamma-ray bursts. The Astrophysical Journal 413, L101-L104 (1993).

2. Nakar, E. Short-hard gamma-ray bursts. Physics Reports 442, 166 - 236 (2007). URL http://wwW.sciencedirect.com/science/article/pii/ S0370157307000476. The Hans Bethe Centennial Volume 1906-2006.

3. Woosley, S. E. \& Bloom, J. S. The Supernova Gamma-Ray Burst Connection. ARA\&A 44, 507-556 (2006). astro-ph/ 0609142.

4. Galama, T. J. et al. An unusual supernova in the error box of the $\gamma$-ray burst of 25 april 1998. Nature 395, 670-672 (1998).

5. Coulter, D. A. et al. Swope Supernova Survey 2017a (SSS17a), the optical counterpart to a gravitational wave source. Science 358, 1556-1558 (2017).

6. Goldstein, A. et al. An ordinary short gamma-ray burst with extraordinary implications: Fermi-gbm detection of grb 170817a. The Astrophysical Journal Letters 848, L14 (2017).

7. Antonelli, L. A. et al. Grb 090426: the farthest short gamma-ray burst? Astronomy \& Astrophysics 507, L45-L48 (2009).

8. Gal-Yam, A., Fox, D. \& MacFayden, A. A novel explosive process is required for the $\gamma$-ray burst grb 060614. Nature 1053-1055 (2006). URL https : / / doi . org/10.1038/ nature05373.

9. Bromberg, O., Nakar, E., Piran, T. \& Sari, R. Short versus long and collapsars versus non-collapsars: A quantitative classification of gamma-ray bursts. The Astrophysical Journal 764, 179 (2013). URL http://stacks.iop.org/0004-637X/764/i=2/a=179.

10. Bellm, E. C. et al. The zwicky transient facility: system overview, performance, and first results. Publications of the Astronomical Society of the Pacific 131, 018002 (2018).

11. D'Ai, A. et al. GRB 200826A: Swift-XRT afterglow detection. GRB Coordinates Network 28300, 1 (2020).

12. Alexander, K. D., Fong, W., Paterson, K. \& Rastinejad, J. GRB 200826A: VLA Detection at 6 GHz. GRB Coordinates Network 28302, 1 (2020). 
13. Chambers, K. C. et al. The Pan-STARRS1 Surveys. arXiv e-prints arXiv:1612.05560 (2016). 1612.05560 .

14. Dey, A. et al. Overview of the DESI legacy imaging surveys. The Astronomical Journal 157, 168 (2019). URL https : / / doi .org/10.38472F1538-38812Fab089d.

15. Leibler, C. N. \& Berger, E. The Stellar Ages and Masses of Short Gamma-ray Burst Host Galaxies: Investigating the Progenitor Delay Time Distribution and the Role of Mass and Star Formation in the Short Gamma-ray Burst Rate. ApJ 725, 1202-1214 (2010). 1009.1147.

16. Fong, W., Berger, E., Margutti, R. \& Zauderer, B. A. A decade of short-duration gamma-ray burst broadband afterglows: Energetics, circumburst densities, and jet opening angles. The Astrophysical Journal 815, 102 (2015). URL http: / / stacks.iop.org/0004-637X/ $815 / i=2 / a=102$.

17. Sari, R., Piran, T. \& Narayan, R. Spectra and Light Curves of Gamma-Ray Burst Afterglows. ApJ 497, L17-L20 (1998). astro-ph/ 9712005.

18. Shivvers, I. \& Berger, E. A beaming-independent estimate of the energy distribution of long gamma-ray bursts: initial results and future prospects. The Astrophysical Journal 734, 58 (2011).

19. Panaitescu, A. \& Kumar, P. Properties of relativistic jets in gamma-ray burst afterglows. The Astrophysical Journal 571, 779 (2002).

20. Dietrich, T. et al. New constraints on the supranuclear equation of state and the hubble constant from nuclear physics - multi-messenger astronomy. Science 370, 1450-1453 (2020). 2002 . 11355.

21. Gompertz, B. P. et al. The Diversity of Kilonova Emission in Short Gamma-Ray Bursts. ApJ 860, 62 (2018). 1710.05442.

22. Clocchiatti, A., Suntzeff, N. B., Covarrubias, R. \& Candia, P. The ultimate light curve of sn 1998bw/grb 980425. The Astronomical Journal 141, 163 (2011).

23. Cano, Z., Wang, S.-Q., Dai, Z.-G. \& Wu, X.-F. The observer's guide to the gamma-ray burst supernova connection. Advances in Astronomy 2017 (2017). 
24. Sobacchi, E., Granot, J., Bromberg, O. \& Sormani, M. A common central engine for long gamma-ray bursts and type ib/c supernovae. Monthly Notices of the Royal Astronomical Society 472, 616-627 (2017).

25. Zhang B.-B., et al. (in prep).

26. Soderberg, A. M. et al. Relativistic ejecta from X-ray flash XRF 060218 and the rate of cosmic explosions. Nature 442, 1014-1017 (2006). astro-ph/ 0604389.

27. Nakar, E. A Unified Picture for Low-luminosity and Long Gamma-Ray Bursts Based on the Extended Progenitor of llGRB 060218/SN 2006aj. ApJ 807, 172 (2015). 1503 . 00441.

28. Graham, J. F. \& Schady, P. The Absolute Rate of LGRB Formation. ApJ 823, 154 (2016). 1511.01466 .

29. Gehrels, N. et al. The Swift Gamma-Ray Burst Mission. ApJ 611, 1005-1020 (2004). astro-ph/0405233.

30. Piran, T., Bromberg, O., Nakar, E. \& Sari, R. The long, the short and the weak: the origin of gamma-ray bursts. Philosophical Transactions of the Royal Society of London Series A 371, 20120273-20120273 (2013). 1206.0700.

Acknowledgements This work was supported by the GROWTH (Global Relay of Observatories Watching Transients Happen) project funded by the National Science Foundation under PIRE Grant No 1545949. GROWTH is a collaborative project among California Institute of Technology (USA), University of Maryland College Park (USA), University of Wisconsin Milwaukee (USA), Texas Tech University (USA), San Diego State University (USA), University of Washington (USA), Los Alamos National Laboratory (USA), Tokyo Institute of Technology (Japan), National Central University (Taiwan), Indian Institute of Astrophysics (India), Indian Institute of Technology Bombay (India), Weizmann Institute of Science (Israel), The Oskar Klein Centre at Stockholm University (Sweden), Humboldt University (Germany), Liverpool John Moores University (UK) and University of Sydney (Australia).

Based on observations obtained with the Samuel Oschin Telescope 48-inch and the 60-inch Telescope at the Palomar Observatory as part of the Zwicky Transient Facility project. ZTF is supported by the National Science Foundation under Grant No. AST-1440341 and a collaboration including Caltech, IPAC, the Weizmann Institute for Science, the Oskar Klein Center at Stockholm University, the University of Maryland, the University of Washington (UW), Deutsches Elektronen-Synchrotron and Humboldt University, 
Los Alamos National Laboratories, the TANGO Consortium of Taiwan, the University of Wisconsin at Milwaukee, and Lawrence Berkeley National Laboratories. Operations are conducted by Caltech Optical Observatories, IPAC, and UW. The work is partly based on the observations made with the Gran Telescopio Canarias (GTC), installed in the Spanish Observatorio del Roque de los Muchachos of the Instituto de Astrofisica de Canarias, in the island of La Palma.

The material is based upon work supported by NASA under award number 80GSFC17M0002.

AJCT acknowledges all co-Is of the GTC proposal and the financial support from the State Agency for Research of the Spanish MCIU through the "Center of Excellence Severo Ochoa" award to the Instituto de Astrofísica de Andalucía (SEV-2017-0709).

The ZTF forced-photometry service was funded under the Heising-Simons Foundation grant \#12540303 (PI: Graham).

S.McB. and J.M. acknowledge support from Science Foundation Ireland under grant number 17/CDA/4723. R.D. acknowledges support from the Irish Research Council (IRC) under grant GOIPG/2019/2033

Analysis was performed on the YORP cluster administered by the Center for Theory and Computation, part of the Department of Astronomy at the University of Maryland.

Resources supporting this work were provided by the NASA High-End Computing (HEC) Program through the NASA Advanced Supercomputing (NAS) Division at Ames Research Center.

These results also made use of Lowell Observatory's Lowell Discovery Telescope (LDT), formerly the Discovery Channel Telescope. Lowell operates the LDT in partnership with Boston University, Northern Arizona University, the University of Maryland, and the University of Toledo. Partial support of the LDT was provided by Discovery Communications. LMI was built by Lowell Observatory using funds from the National Science Foundation (AST-1005313).

M. W. Coughlin acknowledges support from the National Science Foundation with grant number PHY-2010970. S. Anand gratefully acknowledges support from the GROWTH PIRE grant (1545949). Part of this research was carried out at the Jet Propulsion Laboratory, California Institute of Technology, under a contract with the National Aeronautics and Space Administration. E.C. Kool acknowledges support from the G.R.E.A.T research environment and the Wenner-Gren Foundations. P. T. H. P. is supported by the research program of the Netherlands Organization for Scientific Research (NWO). H. Kumar thanks the LSSTC Data Science Fellowship Program, which is funded by LSSTC, NSF Cybertraining Grant \#1829740, the Brinson Foundation, and the Moore Foundation; his participation in the program has benefited this work. S.McB. and J.M. acknowledge support from Science Foundation Ireland under grant number 17/CDA/4723. RD acknowledges support from the Irish Research Council (IRC) under grant GOIPG/2019/2033. P.C. acknowledges support from the Department of Science and Technology via Swarana Jayanti Fellowship award (file no.DST/SJF/PSA-01/2014-15). 
We thank the staff of the GMRT that made these observations possible. GMRT is run by the National Centre for Radio Astrophysics of the Tata Institute of Fundamental Research. We thank D. Bhattacharya, A. Vibhute, and V. Shenoy for help with CZTI analysis.

Competing Interests The authors declare that they have no competing financial interests.

Contributions TA and LPS were the primary authors of the manuscript. MMK is the PI of GROWTH and the ZTF EM-GW program, and SBC is PI of the SGRB program. MC, SA, IA, and MA support development of the GROWTH ToO Marshal and associated program. HK and CF led the reductions of the Gemini data. EB led analysis of the Fermi gamma-ray data. GR, VC, TD, and PTHP contributed to the afterglow, KN, and SN modeling. RD and JM were the GBM burst advocates and provided gamma-ray analysis. DSS, DF, $\mathrm{KH}, \mathrm{AR}$ and AT performed IPN and Konus analyses. AJCT, AV and SBP provided the GTC spectrum. KD performed the WIRC data reduction. PC and SP provided GMRT data. PG, SD and ET provided the LDT data.EH performed galaxy and SED fitting. SI and VB performed the Astrosat analyses. CC contributed to the GROWTH Marshal. BB, AG, DP, AYQH, VK, EK RS, SR, AS, RS contributed to candidate scanning, vetting, and classification. EB, DAD, MJG, RRL, SRK, FJM, AM, PR, BR, DLS, RS, MS, and RW are ZTF builders. All authors contributed to edits to the manuscript.

Correspondence Correspondence and requests for materials should be addressed to Tomás Ahumada (email: tahumada@astro.umd.edu). 


\section{Methods}

\section{Discovery}

Background. The traditional paradigm of classifying a GRB based only on its gamma-ray properties is debated ${ }^{9}$. Although the two types of progenitors broadly map to the time duration of the signals, there is no clear boundary in the bimodal distribution. Some SGRBs show afterglow and host properties akin to LGRBs, e.g. Ref. 734; some LGRBs show no evidence for collapsars to deep limits akin to SGRBs, e.g. Ref. ${ }^{35,36}$.

In the mid 2000s, SGRB-related breakthroughs triggered by the Neil Gehrels Swift Observatory ${ }^{29}$ and HETE-II ${ }^{38}$ included detections of SGRBs X-ray afterglows, identification of likely host galaxies ${ }^{39}$, and the first SGRB optical afterglow ${ }^{40,41}$. Yet, in the first decade of optical follow-up of Swift SGRBs, only about 30 optical/NIR afterglows were detected ${ }^{16}$ and even fewer had associated redshifts. On 2017 August 17, the joint detection of the binary neutron star (BNS) merger (GW170817) in GWs with LIGO and Virgo ${ }^{44}$, and in gamma-rays by Fermi GBM $^{45}$ and INTEGRAL ${ }^{46-48}$, unequivocally confirmed BNS mergers as at least one of the mechanisms that can produce a SGRB. The merger illuminated the entire electromagnetic spectrum ${ }^{49}$ and the optical/NIR emission provided robust evidence of a radioactively powered $\mathrm{KN}^{50-59}$.

The bulk of our knowledge about SGRBs comes from the well-studied Swift SGRB afterglow sample; however, the low Swift local rate of $\mathrm{SGRBs}^{60}$ has hampered the detection of a GW170817-like event. On the other hand, the Fermi GBM is arguably the most prolific engine of discovery for compact binary mergers, as it detects $\approx 1$ SGRB each week, which is four times the rate of Swift $t^{61,62}$, and comparable to the rate of the LIGO and Virgo detectors when in observing mode $\left(\approx 1.5\right.$ per week during $\left.\mathrm{O}_{3} \mathrm{a}^{63}\right)$. Unfortunately, there is a relative paucity of electromagnetic observations of Fermi GRBs and LIGO/Virgo GW events alike because it is challenging to pinpoint them within their positional uncertainties of tens to thousands of square degrees ${ }^{64-66}$.

In order to better understand the phenomenology of GRBs and compact binary mergers, wide field of view (FOV) optical instruments have looked for Fermi GRBs and LIGO/Virgo GW counterparts. In 2013, the Palomar Transient Factory (PTF) ${ }^{67}$ used a $7 \mathrm{deg}^{2}$ camera on the Palomar 48 inch Oschin telescope (P48) to discover the first optical afterglow of a GRB based solely on a Fermi GBM localization ${ }^{68}$ and subsequently found afterglows of 7 other LGRBs ${ }^{69}$. Now, the Zwicky Transient Facility $\left(\mathrm{ZTF}^{10} 71,72\right)$, a $47 \mathrm{deg}^{2}$ camera mounted at the P48 telescope, has 
enabled searches an order of magnitude faster in areal and volumetric survey speed and has been used to search the coarse error regions of Fermi GBM SGRBs ${ }^{73}$ and LIGO/Virgo events ${ }^{74-77}$. Both PTF and ZTF have also discovered afterglow-like transients with optical, X-ray, and radio emission, but no gamma-ray counterpart ${ }^{78-80}$.

Gamma-ray detections. Fermi (GBM trigger 620108997) ${ }^{81}$, and the IPN instruments - AGILE ${ }^{82}$, INTEGRAL (SPI-ACS), Mars-Odyssey (HEND), Konus-Wind ${ }^{83}$, and AstroSat ${ }^{84}$ detected the burst. The GBM localization calculated by the ground software ${ }^{85,86}$ is RA $=4.7$, Dec $=35.3$ (J2000 degrees, equivalent to $\mathrm{J} 2000 \mathrm{00h} 18 \mathrm{~m}, 35 \mathrm{~d} 17^{\prime}$ ) with a statistical uncertainty of 1.7 degrees $^{81}$. The BALROG localization is consistent with this ${ }^{87}$. The final GBM localization of the GRB was available a few minutes after the trigger, and it enclosed 339 (63) deg $^{2}$ within the 95\% (50\%) credible region.

The light curve shows a bright GRB featuring three distinct peaks in the 50-300 keV energy range, with with a signal-to-noise ratio $(\mathrm{S} / \mathrm{N})$ of $\sim 100$ and a $T_{90}$ duration of $1.14 \pm 0.13 \mathrm{~s}$. This GRB is within the top 36\% in terms of fluence (in the 50-300 keV energy band as measured over $T_{100}$ ) from bursts recorded in the Fermi GBM catalog ${ }^{62}$. Spectral analysis was performed using the RMFIT 4.4.2 light curve and spectral analysis software ${ }^{a}$ in the interval of $T_{0}$ to $T_{0}+1.152 \mathrm{~s}$ encompassing the $T_{90}$ start time and duration. The detector selection for the analysis consisted of the brightest NaI detectors with angle $<60^{\circ}$ (N6, N7, N8, N9, NB11) and BGO detector B1. $\mathrm{NaI}$ and BGO detector data from $\sim 8 \mathrm{keV}$ to $\sim 900 \mathrm{keV}$ and $\sim 300 \mathrm{keV}$ to $\sim 40 \mathrm{MeV}$ respectively was used for the analysis. The Band Model ${ }^{88}$, a standard model for GRB analysis ${ }^{89}$, provided best fit with parameters consistent with the initial analysis ${ }^{90}$, where $E_{\text {peak }}=88.9 \pm 3.2 \mathrm{keV}, \alpha$ $=-0.26 \pm 0.07$, and $\beta=-2.4 \pm 0.1$. The peak flux in the $64 \mathrm{~ms}$ time $(10 \mathrm{keV}-1 \mathrm{MeV})$, measured from $T_{0}+1.152 \mathrm{~s}$, is $64.3 \pm 2.1 \mathrm{ph} \mathrm{cm}^{-2} \mathrm{~s}^{-1}$. The fluence over $T_{90}(10 \mathrm{keV}$ to $1 \mathrm{MeV})$ is $42.6 \pm 0.2 \times 10^{-7} \mathrm{erg} \mathrm{cm}^{-2}$ (the fit merit of the spectral fit is 1.09).

A time resolved spectral analysis was performed from $T_{0}$ to $T_{0}+1.152 \mathrm{~s}$. The data was binned by $\mathrm{S} / \mathrm{N}$, with at least $20 \sigma$ for the $\mathrm{S} / \mathrm{N}$ in each bin. For 6 of the bins, the best-fit parameters of the Band Model are displayed in Extended Data Table 5. The parameters for the Band model in the initial time interval were poorly constrained so a cutoff power law (Comptonised) model ${ }^{91}$ was fitted to this interval. The Band Model was primarily used as this was the best constrained model

${ }^{a}$ Available at the Fermi Science Support Center: https://fermi.gsfc.nasa.gov/ssc/data/p7rep/ analysis/rmfit/ 
for the time integrated analysis. The Castor C-stat statistic ${ }^{92}$ was used for its robustness when dealing with low count statistics, while the background was modeled as a first order polynomial over all qualifying detectors, as discussed above, using TTE data products. Spectral evolution of the $E_{\text {peak }}$ parameter is observed over the duration of the burst, and is found to exhibit hardness-intensity tracking behavior ${ }^{91,92}$.

The distribution of GRB $T_{90}$ durations demonstrates a bimodality that is best fit with double log-normal components. This suggests two distinct but overlapping progenitor probabilities, and by extension, classifications for short and long GRBs. Using only the $T_{90}$, and the parameters derived for the Fermi GBM distribution of bursts in Ref. 9 , we determine a SGRB probability of $64.94 \%_{-11.30}^{11.59}$ for GRB 200826A.

According to BATSE ${ }^{93}$, the overlap of GRB $T_{90}$ duration distributions occurs at $\sim 2 \mathrm{~s}$. In the past, GRB hardness ratios, quantifiable for the majority of GRBs in the absence of location information, have been used as a measure for the spectral hardness. Hardness ratio is presented for the GBM catalogue events in Ref. ${ }^{62}$, where the data shows an anti-correlation between hardness and duration; however, there is a large scatter in the data. We derive a $T_{90}$ value of $4.2 \mathrm{~s}$ as the duration where an event has equal probability of being in the short or long class ${ }^{62}$ based on 2353 GRBs. We choose the $E_{\text {peak }}$ parameter instead of hardness for separating LGRB and SGRB populations as it does not require set energy bands for a comparison to be made ${ }^{94}$. In Figure 1 we presents a log-log plot of the $T_{90}$ and $E_{\text {peak }}$ parameter, for which we fit two log-normal distributions, one for each class, and the probability that an event is an SGRB is translated into a colour. From this analysis, the probability that GRB 200826A belongs to the short class is $74 \%{ }^{95}$.

GRB 200826A triggered Konus-Wind $(\mathrm{KW})$ at $T_{0 \mathrm{KW}}=16195.106 \mathrm{~s}$ UT (04:29:55.106). The propagation delay from Earth to Wind is $2.540 \mathrm{~s}$ for this GRB; correcting for this factor, the KW trigger time corresponds to the Earth-crossing time 16192.566 s UT (04:29:52.566). The burst light curve shows a multi-peaked pulse which starts at about $T_{0 \mathrm{KW}}-0.10 \mathrm{~s}$ and has $T_{100}=0.97 \mathrm{~s}$, $T_{90}=0.67_{-0.03}^{+0.13} \mathrm{~s}$, and $T_{50}=0.28_{-0.03}^{+0.04} \mathrm{~s}$ measured in the $80-1300 \mathrm{keV}$ band. Considering only the duration, the $T_{90}$ and $T_{50}$ of GRB 200826A are consistent with the short GRB population in the KW sample. The $T_{90}$ and $T_{50}$ durations at which a KW-detected GRB has an equal probability of being short- or long-duration are $2 \mathrm{~s}$ and $0.7 \mathrm{~s}$, respectively ${ }^{96}$. The spectral lag between the 20-80 keV and 80-330 keV $16 \mathrm{~ms}$ light curves is $30 \pm 11 \mathrm{~ms}$, consistent with the bulk of KW short GRBs ${ }^{97}$. 
During the burst, KW measured five spectra in the $20 \mathrm{keV}-10 \mathrm{MeV}$ band. The first four with $64 \mathrm{~ms}$ accumulation time cover the interval from $T_{0 \mathrm{KW}}$ to $T_{0 \mathrm{KW}}+0.256 \mathrm{~s}$ and the fifth, from $T_{0 \mathrm{KW}}+0.256 \mathrm{~s}$ to $T_{0 \mathrm{KW}}+8.448 \mathrm{~s}$. The time-averaged spectrum of the burst (measured from $T_{0 \mathrm{KW}} \mathrm{s}$ to $T_{0 \mathrm{KW}}+8.448 \mathrm{~s}$ ) is best fit in the $20 \mathrm{keV}-2 \mathrm{MeV}$ range by the Band function with a low-energy photon index $\alpha=1.26_{-1.12}^{+1.91}$, a high-energy photon index $\beta=-2.32_{-0.15}^{+0.12}$, and a spectrum peak energy $E_{\text {peak }}=67_{-11}^{+13} \mathrm{keV}\left(\chi^{2} /\right.$ d.o.f. $\left.=50 / 59\right)$. The burst had a fluence of $4.60_{-0.60}^{+0.71} \times 10^{-6} \mathrm{erg} \mathrm{cm}^{-2}$, and a 16-ms peak flux, measured from $T_{0 \mathrm{KW}}+0.544 \mathrm{~s}$, of $9.81_{-1.64}^{+1.83} \times$ $10^{-6} \mathrm{erg} \mathrm{cm}^{-2} \mathrm{~s}^{-1}$ (both in the $20 \mathrm{keV}-10 \mathrm{MeV}$ energy range). Using the spectroscopic redshift $z=0.7481^{98}$ we have estimated the following rest-frame parameters: the isotropic energy release $E_{i s o}$ is $7.17_{-0.94}^{+1.11} \times 10^{51} \mathrm{erg}$, the peak luminosity $L_{i s o}$ is $2.67_{-0.45}^{+0.50} \times 10^{52} \mathrm{erg} \mathrm{s}^{-1}$, and the rest-frame peak energy of the time-integrated spectrum, $E_{\text {peak,z }}$ is $117_{-19}^{+23} \mathrm{keV}$.

With these values, GRB 200826A is within the softest $~ 1 \%$ of $\mathrm{KW}$ short GRBs in terms of the observed $E_{\text {peak,z }}$ and is within the $1 \sigma$ prediction band of both the 'Amati' and 'Yonetoku' relations based on 315 long/soft (Type II) GRBs with known z ${ }^{99-101}$. Furthermore, in the $E_{\text {iso }}-E_{\text {peak,z }}$ plane, GRB 200826A is inconsistent with the short-hard (Type I) GRB population (see Extended Data Figure 1). Thus, despite the short duration, the KW parameters of GRB 200826A imply that it belongs to the long/soft GRB population.

In addition to the IPN detections, the Cadmium Zinc Telluride Imager $\left(\mathrm{CZTI}^{102}\right)$ on board AstroSat also detected the burst. We reanalysed the data, combining data from all four quadrants to create $20-200 \mathrm{keV}$ light curves with $0.05 \mathrm{~s}, 0.1 \mathrm{~s}$ and $0.2 \mathrm{~s}$ bins. All light curves show a single pulse, with hints of sub-structure in during the rise in the smaller time bins (see Extended Data Figure 1). We process the $0.05 \mathrm{~s}$ light curves with the CIFT pipeline ${ }^{103}$, which incorporates better data analysis as compared to the quick-look pipeline and produces more robust results. Our reanalysis yields a peak time of UT 04:29:52.95 - consistent with the Fermi peak. The new value of $T_{90}$ is $0.94_{-0.18}^{+0.72} \mathrm{~s}$, significantly shorter than the quick-look values reported in Ref. ${ }^{84}$, but consistent with Fermi and Konus-Wind.

Optical. We ingested the GBM localization map into the GROWTH Target-of-Opportunity (ToO) marshal, an interactive tool design to plan and schedule ToO observations for $\mathrm{ZTF}^{73}$. The observation plan generated by the ToO marshal relies on gwemopt ${ }^{104-106}$, a code that optimizes the telescope scheduling process for skymaps with a healpix format, like the Fermi-GBM maps. The gwemopt procedure involves slicing the skymap into predefined tiles of the size and shape of the ZTF 
field-of-view, determining which fields have the highest enclosed probability, and optimizing observations based on airmass and visibility windows. For this purpose, we used a modified version of the "greedy" algorithm described in Ref. ${ }^{107}$, implemented within gwemopt. The resulting optimized plan for the optical follow-up of GRB 200826A consisted of four primary ZTF fields and one secondary field ${ }^{b}$. The fields were observed in the $r$ - and $g$-band for $300 \mathrm{~s}$ each, starting 4.9 hours after the GBM detection. The observing plan for the first night covered $186 \mathrm{deg}^{2}$, corresponding to $77 \%$ of the GBM region (see Fig 2). Once the Konus-Wind data became available on ground at about 17:55 UT, the 288 arcmin $^{2}$ IPN error box was derived using Konus, GBM, and HEND data, which allowed the X-ray Telescope $\left(\mathrm{XRT}^{108}\right.$ ) on board Swift to initiate a ToO at about 20:45 UT. The IPN box was published later at about 21:30 UT ${ }^{109}$.

Reference images of the fields are then subtracted from the ZTF ToO observations and any high significance difference $(>5 \sigma)$ generates an alert ${ }^{72,110}$ that contains relevant information about the transient. We queried the stream of alerts using three different tools: the GROWTH marshal ${ }^{111}$, the Kowalski infrastructure ${ }^{112 c}$ and AMPEL ${ }^{113-11 d d}$. Our filtering scheme has been described in previous SGRB and GW searches ${ }^{73,76}$, but we summarize the main points here. We aim to identify sources that 1) are spatially coincident with the skymap, 2) are detected only after the GBM trigger, 3) are far from known bright sources, 4) are spatially distinct from Pan-STARRS 1 (PS1 ${ }^{13}$ ) stars (based on Ref. ${ }^{118}$ ), 5) have at least two detections separated by at least 15 minutes to avoid moving objects, 6) have a real-bogus score $\left(\mathrm{RB}^{112,119}\right)$ greater than 0.15 , and 7 ) showed an increase in their flux relative to the reference image. For each candidates passing these filters, we visually inspected the light curve, cross-matched with the PS1-DR2 catalog to check for previous activity, and cross-matched against the Wide-field Infrared Survey Explorer (WISE ${ }^{120}$ ) catalog to determine whether it was consistent with an active galactic nucleus (AGN) based on its position in the WISE color space ${ }^{121-123}$. Additionally, we searched the Minor Planet Center (MPC) to ensure that our potential counterparts were not consistent with known solar system objects. Forced photometry ${ }^{124}$ was performed using data from the 10 nights previous to the trigger, to remove young $\mathrm{SNe}$ from the sample. A final quality check was done by querying for alerts around each candidate, and rejecting transients that have multiple alerts within a radius of 15 " as these alerts could suggest artifacts, ghosts, or slow-moving objects near the stationary points ${ }^{125}$.

\footnotetext{
${ }^{b}$ The ZTF secondary fields are strategically located to cover the chip-gaps of the fields in the primary grid "https://github.com/dmitryduev/kowalski

$a$ https://github.com/AmpelProject
} 
After the first night of observations 28195 alerts were generated in the region and 14 sources passed every stage of our filtering criteria ${ }^{126}$. The SGRB afterglow, ZTF20abwysqy ${ }^{127}$, was not in our first selection as there was a ZTF alert 11" NE; however, it passed all the other filtering criteria. Two of the ZTF ToO fields triggered during the first night covered $>99 \%$ of the triangulated IPN region. However, none of the candidates reported in Ref. ${ }^{126}$ fell there. For our second night of observations, we scheduled $600 \mathrm{~s}$ observations in $r$ - and $g$-band and queried the database.

Probability of Chance Coincidence. We roughly calculate the probability of chance coincidence $(p)$ of this optical transient to be independent of GRB 200826A. For this we follow Ref. ${ }^{128}$ and use Poissonian statistics to derive the probability for one or more events to be randomly coincident:

$$
p=1-e^{-\lambda}
$$

where $\lambda$ is the product of three different values, $\lambda=\prod_{i=1}^{3} r_{i}$. For this study, we consider $r_{1}$ to be the time window between the last ZTF non-detection ( 0.72 days before the trigger time) and the time of the detection ( 0.21 days, see Extended Data Table 1$)$, resulting in $r_{1}=0.93$ days. The parameter $r_{2}$ is the rate of Fermi GRBs, which from the latest GBM catalog ${ }^{62}$ gives $\sim 0.65$ bursts per day. Finally $r_{3}$ is the ratio between the total IPN area $\left(288 \operatorname{arcmin}^{2}\right)$ and the sky. This derivation gives a $p=1.16 e-6$, and allows us to rule out a random association between the GRB and the afterglow at the $4.87 \sigma$ level.

\section{Follow-up}

The summary of the follow-up results can be found in Extended Data Table 1Extended Data Table 3. and Extended Data Table 4 .

Optical/NIR. Here we present our optical and near infrared (NIR) follow-up results. In addition to our observations, MASTER ${ }^{129}$ and Kitab follow-up with a clear filter ${ }^{130,131}$ led to upper limits of $18.3 \mathrm{mag}$ and $20.4 \mathrm{mag}$ respectively.

Las Cumbres Observatory. We performed follow-up of ZTF20abwysqy with the Spectral camera mounted at the 2-m Faulkes Telescope North (FTN), located at Haleakala Observatory. Starting on 2020-08-28 11:32:26 UT we acquired three sets of images with 300s exposures each in $g$ - and $r$-bands through the LCO observation portal Furthermore, we obtained a second

\footnotetext{
https://observe.lco.global/
} 
(reference) epoch in $g$ - and $r$-bands on 2020-09-02 UT. Our images were reduced by an automatic subtraction pipeline. Our pipeline retrieves images from LCO, stacks them, extracts sources from the image using Source Extractor ${ }^{132}$ and performs photometric calibration of sources using the PS1 catalog. Then, the pipeline performs image subtraction using the High Order Transform of Psf ANd Template Subtraction code (HOTPANTS ${ }^{133}$ ) to subtract a PSF-scaled reference image aligned using $\mathrm{SCAMP}^{134}$. Our photometry measurements reported in $g$ - and $r$-band were determined after host subtraction using the LCO reference images in the same filters acquired on 2020-09-05.

Hale Telescope. We obtained two epochs of dithered J-band imaging with the Wide Field Infrared Camera (WIRC ${ }^{135}$ ) on the Palomar Hale 200-in (P200) telescope on 2020-08-28 UT and on 2020-09-04 UT, spending an hour integrating on target during each epoch. We reduced the images using the image reduction pipeline described in Ref. ${ }^{136}$. Images were aligned and stacked using SWarp ${ }^{137}$ and calibrated against the 2MASS catalog. Image subtraction between the two epochs was performed using the method described in Ref. ${ }^{138}$ to derive flux measurements and its uncertainty for the first epoch.

Gran Telescopio Canarias. The 10.4m Gran Telescopio Canarias (GTC) (+OSIRIS) obtained spectroscopic observations starting on August 30, 04:30 UT. Two 1200s exposures were gathered with the R1000B grism and one 1200s exposure was obtained with the R2500I grism, in order to cover the entire 3700-10000 A range. The slit was placed covering the position of the potential host galaxy. Standard routines from the Image Reduction and Analysis Facility (IRAF) were used to reduce the data.

Lowell Discovery Telescope. We used the Large Monolithic Imager (LMI ${ }^{139}$ ) mounted on the $4.3 \mathrm{~m}$ Lowell Discovery Telescope (LDT) to observe the optical transient ZTF20abwysqy on three different nights: August 29, September 13, and September 19 (3.2, 18.1 and 24.2 days after the GRB trigger). Observations were conducted with an average airmass of 1.0 while the seeing varied from 1.1" for the first night to $1.4^{\prime \prime}$ and $1.6^{\prime \prime}$ for the second and third night, respectively. We took 8 exposures of $180 \mathrm{~s}$ in the $r$-band on August 29. Images were taken with different filters on September 13: 5 exposures of $180 \mathrm{~s}$ in $u$-band, 4 exposures of $180 \mathrm{~s}$ in $g$-band, 6 exposures of $180 \mathrm{~s}$ in $i$-band and 6 exposures of $180 \mathrm{~s}$ in z-band. Finally, 10 exposure of $150 \mathrm{~s}$ in the $i$-band and $r$-band were taken during the last night of observations on September 19. We used standard procedures to perform bias and flat-field correction. The astrometry was calibrated against the SDSS catalog (release DR16 ${ }^{140}$ ) and frames were aligned using SCAMP and stacked with SWarp. 
After stacking the images, we extracted sources using Source Extractor and the magnitudes were calibrated against 45 PS1 stars in the field on average. We used HOTPANTS to perform image subtraction between the first and third epochs and found a source in the $r$-band at the location of ZTF20abwysky with a magnitude of $24.46 \pm 0.12 \mathrm{mag}$ (see Extended Data Table 1). We determine the magnitudes of the host galaxy using the second epoch of observations. To verify this result, we used the 80 days $i$-band GMOS-North (see description in the paragraph below) as a reference and the HOTPANTS subtraction shows a source with $r$-band magnitude of $24.76 \pm 0.23 \mathrm{mag}$, consistent with the result using the LDT reference.

Gemini Observatory. We acquired images of the transient location on September 23, October 10, and November 7 corresponding to $28.28,46.15$ and 80.23 days after the GRB trigger respectively, which we denote as epoch 1, 2, and 3. We used GMOS-North, mounted on the Gemini North 8-meter telescope on Mauna Kea, under the approved Director's Discretionary Time (DDT) proposal DD-104 (P.I.: L. Singer). The host galaxy coordinates, in the Gemini images, are $\alpha=00^{d} 27^{m} 08.5557^{s}$, $\delta=+34^{d} 01^{m} 38.634^{s}$.

The first set of observations (epoch 1) was scheduled to be closest in time to a SN1998bw-like peak without suffering from moon illumination. All three sets of observations consisted of $14200 \mathrm{~s}$ $r$ - and $i$-band exposures, with a position angle of $45^{\circ}$ to avoid blooming from neighboring bright stars. The average airmass for the observations was $\sim 1.0$ and the seeing was stable throughout the three epochs, at $0.7^{\prime \prime}, 0.5^{\prime \prime}$, and $0.85^{\prime \prime}$. The images were later reduced using DRAGONSf ${ }^{41}$, a Python-based data reduction platform provided by the Gemini Observatory.

We extracted sources using Source Extractor and determined a photometric zero point using 23 PS1 stars in the field. Using the ZOGY ${ }^{142}$ algorithm-based python pipeline, we performed image differencing on Gemini data. The pipeline makes use of Source Extractor, PSFEx ${ }^{143}$, SWarp, SCAMP, and PyZOGY ${ }^{144}$ to perform image subtraction. We took a 1300 pix $\times 1300$ pix sized cutout for both the science and reference images, centered at the ZTF20abzwysqy position in the image in order to achieve good subtraction quality, as the images were affected by background variation. Sources were extracted using Source Extractor from both science and reference images with a 5- $\sigma$ detection threshold. The resulting catalogues were fed to SCAMP, which calculates and corrects for the astrometric errors in both science and reference catalogues with the help of a Gaia data release 2 catalogue ${ }^{145}$ for the same field. Using SWarp, images are

${ }^{f}$ https://dragons.readthedocs.io/ 
then re-sampled to subtract the background and the point-spread function (PSF) of the images. Source Extractor-generated weight maps were used as input to the SWarp to generate variances, which when added with the images' Poisson noise in quadrature, which results in the rms image. We select good sources from Source Extractor catalogues based on their S/N, full width at half-maximum (FWHM) and Source Extractor flag values. Sources which raised a Source Extractor flag were discarded. These sources were used to match the flux level of the images. We used PSFEx to extract the PSF model of the re-sampled images with the Source Extractor catalogue as input to PSFEx. The rms images, re-sampled images, PSF models and astrometric uncertainty are used as input to PYZOGY, which generate the difference image and a corrected score image as final products ${ }^{142}$. A source is detected with a ZOGY corrected score of 4 in the $i$-band, at the location of the transient. With ZOGY, we derive an $i$-band magnitude of $25.49 \pm 0.12 \mathrm{mag}$ for epoch 1 . No source is detected in the r-band of epoch 1 nor in any band in epoch 2.

We have confirmed the ZOGY-based results using an independent image subtraction pipeline, FPipe $^{146}$, which is based on empirically measuring the PSFs of the science and reference images and matching them using the common PSF method $\left(\mathrm{CPM}^{147}\right)$. We detect the source with an $i$-band brightness of $25.45 \pm 15 \mathrm{mag}$ in epoch 1 . The results agree within uncertainties with the ZOGY-based subtractions. We do not detect the source in the $r$-band up to a 5- $\sigma$ limit of $r>$ $25.6 \mathrm{mag}$ for epoch 1 . No source is detected in either filter during epoch 2 up to a $5 \sigma$ upper limit of $i>25.4$ mag and $r>25.5 \mathrm{mag}^{148}$ (see Figure 3 ).

Therefore, using two independent image subtraction pipelines, we confirm the detection of a source in the $i$-band, at the location of the transient.

UV/X-ray. Swift began observing the IPN localization region of GRB 200826A 0.7 days after the trigger. The last two observations were $\sim 4 \mathrm{ks}$ and triggered as ToO observations. The XRT data was reduced by the the online reduction pipeline ${ }^{849,150}$. As the hardness ratio remains constant within error bars, we assume a single absorbed power law spectrum, with a Galactic neutral hydrogen column ${ }^{151}$ of to $6.02 \times 10^{20} \mathrm{~cm}^{-2}$. We convert the count rates (see Extended Data Table 3p to flux density at an energy of $1 \mathrm{keV}$, using the parameters derived by the Swift pipeline: a photon index of $\Gamma_{X}=1.5_{-0.5}^{+0.7}$, an intrinsic host absorption of $n_{H, i n t}=6_{-6}^{+32} \times 10^{20} \mathrm{~cm}^{-2}$, and an unabsorbed counts-to-flux conversion factor of $4.27 \times 10^{-11} \mathrm{erg} \mathrm{cm}^{-2} \mathrm{ct}^{-1}$.

\footnotetext{
$\sqrt[8]{\text { https://www.swift.ac.uk/xrt_curves/00021028/ }}$
} 
The first UV/Optical Telescope (UVOT) observations of ZTF20abwysqy was $~ 1.6$ days after the burst and the detection in the white filter was associated to the underlying galaxy 11 .

Radio. For the afterglow modeling, we additionally use data reported by Ref. 12 . They measure a flux density of $\sim 40 \mu \mathrm{Jy}$ at a mean frequency of $6 \mathrm{GHz}$ using the Karl G. Jansky Very Large Array (VLA), 2.28 days after the trigger. We observed GRB 200826A in band-5 (1050-1450 $\mathrm{MHz}$ ) of the upgraded Giant Metrewave Radio Telescope (uGMRT ${ }^{154}$ ) on 2020-09-09 15:41:30.5 UT and 2020-09-14 23:38:25.5 UT ( 14.5 and $\sim 19.8$ days after the burst respectively) under the approved DDT proposal ddtC147 (P.I.: Poonam Chandra). The data was recorded with the GMRT Wideband Backend (GWB) correlator with a bandwidth of $400 \mathrm{MHz}$ divided in 2048 channels, with the central frequency of $1250 \mathrm{MHz}$. The total on-source time was $\sim 75$ mins with overheads of $\sim 30$ mins. 3C48 was used as the flux and bandpass calibrator and J0029+349 was used as the phase calibrator. We used standard data reduction procedures in Common Astronomy Software Applications (CASA) ${ }^{155}$ for analysing the data. The dead antennas were first flagged by manual inspection and the end channels were flagged due to low gain. The automatic flagging algorithms incorporated into the CASA task flagdata were used to remove most of the Radio Frequency Interference (RFI). Any remaining corrupted data were then flagged manually. The calibrated data was then imaged and self-calibrated to get the final image. The synthesized beam for the final image was $\sim 4^{\prime \prime} \times 2^{\prime \prime}$. We found no evidence of radio emission at the GRB position on both days. The $3 \sigma$ upper limits were $48.6 \mu \mathrm{Jy} / \mathrm{beam}$ for the observations taken on 2020-09-09 156 and 57.4 $\mu \mathrm{Jy} / \mathrm{beam}$ for the observations taken on 2020-09-14.

\section{The Host Galaxy}

Our GTC spectra of the host galaxy show strong [OII] and [OIII] features at $z=0.748$, in agreement with a previous report based on data from the Large Binocular Telescope Observatory $(\mathrm{LBTO})^{98}$.

We modeled the GTC spectrum with the Penalized Pixel-Fitting (pPXF) ${ }^{157}$ to infer the properties of the host galaxy's stellar populations, resulting in a fit with $\chi_{\nu}^{2}=1.011$. The pPXF results confirmed the [OII] features at $3726 \AA$ and [OIII] lines at $4959 \AA$ and $5007 \AA$, indicators of recent star-formation and young, hot stars. The stellar population age derived from the weighted ages

of the templates is $1.514_{-0.85}^{+3.83} \mathrm{Gyr}$, with evidence for a younger $\sim 0.1 \mathrm{Gyr}$ population (see Figure Extended Data Figure 2 and Extended Data Table 6). 
Additionally, we corrected the magnitudes of the host galaxy (see Extended Data Table 2) using foreground extinction maps ${ }^{158}$ and fed them to Prospector ${ }^{159}$, to model the SED of the host galaxy. Prospector uses $\operatorname{fsps}^{160,161}$ to generate multiple stellar populations, and fits the observed photometry to determine the formed mass of the galaxy, age, and intrinsic extinction among other parameters, using the WMAP9 cosmology ${ }^{162}$ internally. We fitted the photometry to a galaxy using the Chabrier ${ }^{163}$ initial mass function (IMF), the Calzetti ${ }^{164}$ extinction curves for the dust around old stars, and a star formation history (SFH) with the form of $t e^{-t / \tau}$. Our results using the nested sampling dynest $\mathrm{y}^{165}$ algorithm, gave a galactic stellar mass distribution of $M_{\text {gal }}=4.64 \pm 1.67 \times 10^{9} M_{\odot}$, a mass-weighted galactic age of $t_{\text {gal }}=1.08_{-0.72}^{+1.28} \mathrm{Gyr}$, a metallicity of $\log \left(Z / Z_{\odot}\right)=-1.06_{-0.48}^{+0.67}$, a dust extinction of $A_{V}=0.34_{-0.22}^{+0.33}$, and a star formation rate (SFR) of $4.01_{-3.59}^{+41.87} M_{\odot} \mathrm{yr}^{-1}$ (see the resulting SED in Extended Data Figure 2 and the posterior probability distributions for the free parameters in Extended Data Figure 3. We derived the SFR and weighted-mass age following similar studies on GRB host galaxies ${ }^{166-168}$, and the stellar mass from the mass fraction derived with Prospector.

\section{Modeling}

For the modeling of the multiwavelength emission, all optical and NIR observations were from difference images and were corrected for foreground extinction ${ }^{158}$.

The afterglow. In the standard synchrotron fireball model a power-law energy distribution characterized by the index $p, N(E) \propto E^{-p}$, results in a SED described by a series of broken power laws ${ }^{17}{ }^{170}$. The frequencies at which the broadband SED presents its breaks are the self-absorption frequency $\nu_{a}$, the synchrotron frequency $\nu_{m}$, and the cooling frequency $\nu_{c}$.

The temporal decline of both the optical and X-ray data during the first four days can be described by a single power law model which suggests the jet-break has not yet occurred. Our ZTF $g$-band observations are the most constraining in the optical therefore we use these to estimate the temporal decline rate $\alpha_{\mathrm{o}}=-1.05 \pm 0.13$. We use the $1 \mathrm{keV}$ XRT data to find $\alpha_{\mathrm{x}}=-0.89 \pm 0.07$. The similar slopes between the optical and X-ray observations suggest the location of the cooling break frequency, $\nu_{c}$, lies beyond X-ray frequencies. Therefore, we estimate the spectral index at $\sim 1$ day between the $\mathrm{X}$-ray and optical as $\beta_{\mathrm{ox}}=-0.67 \pm 0.02$.

We now use $\alpha_{\mathrm{o}}$ and $\alpha_{x}$ to estimate the power law index of the electron energy distribution, $p$, and to determine the circumburst density profile. In a constant density (ISM-like) medium 
$\alpha_{\mathrm{ISM}}=\frac{3(1-p)}{4}\left(\nu<\nu_{c}\right)$, which gives $p_{\mathrm{o}}=2.44 \pm 0.17$ and $p_{\mathrm{x}}=2.17 \pm 0.10^{171}$. In the wind-like scenario $\alpha_{\text {wind }}=\frac{(1-3 p)}{4}$, which gives $p_{\mathrm{o}}=1.78 \pm 0.17$ and $p_{\mathrm{x}}=1.50 \pm 0.10$. The optical to $\mathrm{X}$-ray spectral index $\beta_{\mathrm{ox}}$ gives an estimate for $p$ of $2.34 \pm 0.04\left(\beta=\frac{1-p}{2}\right)$. Theoretical studies of relativistic collisionless shocks predict $p \gtrsim 2$ and particularly $p \sim 2.2$ in the ultra-relativistic limit ${ }^{172}$. Given the low values of $p$ for the wind-like scenario we choose to assume an ISM-like density profile and $p \sim 2.4$ throughout this work.

We note the possibility of a wind-like environment with $p<2$ and a spectral break $\nu_{c}$ between the optical and X-rays. Such a scenario would not change the predicted optical and NIR emission and it would be unable to account for the late GMOS $i$-band detection without an additional component. However, this scenario may allow for a better fit of the afterglow X-ray evolution, which would decay at a rate shallower than the optical by $\Delta \alpha=0.25$. We do not consider this scenario further because of the theoretically disfavored value of $p$.

Bayesian afterglow modeling. We now use Bayesian inference to analyze the X-ray, optical, NIR, and radio counterpart. We use two independent pipelines, one using Markov chain Monte Carlo (MCMC) based on the EMCEE Python package ${ }^{173}$ and one using nested sampling based on PyMultinest ${ }^{174}$. The pipelines use different priors and implementations, but arrive at consistent results. Both utilize the afterglowpy python package ${ }^{175}$ to estimate the physical parameters of the multi-wavelength afterglow. Afterglowpy is a public, open-source computational tool which models forward shock synchrotron emission from relativistic blast waves as a function of jet structure and viewing angle. Descriptions of the MCMC implementation may be found in Refs. ${ }^{176,177}$ and the nested sampling implementation in Ref. ${ }^{20}$.

For this work we assume Gaussian statistics for the optical and radio data, while assuming Poissonian statistics (via the $\mathrm{C}$-statistic ${ }^{179}$ ) for the X-ray data due to low detector counts. The

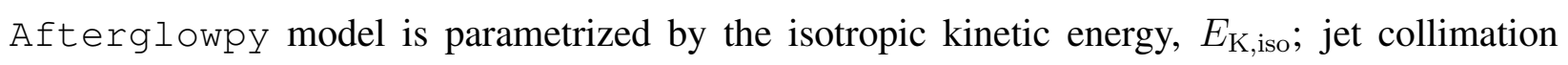
angle, $\theta_{c}$; viewing angle, $\theta_{v}$; the circumburst constant density, $n$; the spectral slope of the electron distribution, $p$; the fraction of energy imparted to both the electrons, $\epsilon_{e}$, and to the magnetic field, $\epsilon_{B}$, by the shock. The redshift and luminosity distance of the source are held fixed.

Our modelling of the host indicates a small galaxy of stellar mass $\sim 5 \times 10^{9} M_{\odot}$ with moderate extinction $A_{V}=0.34_{-0.22}^{+0.33}$ (see $\$ 3$ for details). In the MCMC implementation, we incorporate a Small Magellanic Cloud-like host extinction correction with total-to-selective extinction $R_{V}=2.93^{180}$ implemented with the dust-extinction software package ${ }^{181}$. We leave the 
color excess $E(B-V)$ as a free parameter, with a prior distribution computed from the $A_{V}$ posterior found from modelling the host galaxy. This posterior is similar to the distribution of host extinction values observed in LGRBs with well-sampled multiband photometry ${ }^{182-184}$. The nested sampling implementation performs no extinction correction.

Using a top hat model for the jet structure, we perform a search over the parameter space by allowing all the parameters $E_{\mathrm{K} \text {,iso }}, \theta_{c}, \theta_{v}, n, p, \epsilon_{e}, \epsilon_{B}$, and $E(B-V)$ to vary with broad priors. We report only the MCMC results, although the nested sampling results are consistent. Given both the highly degenerate nature of the afterglow fitting and the low number of observations available, some of the parameters are not particularly constrained. One exception is the spectral slope of the electron distribution, for which we find $p=2.4 \pm 0.04$, consistent with the analytical results. The uncertainty in the circumburst density, $n=5.5_{-5.4}^{+187.3} \times 10^{-2} \mathrm{~cm}^{-3}$, includes typical ranges for both SGRBs and LGRBs. We find very little reddening from the host, $E(B-V)=2.5_{-2.3}^{+4.8} \times 10^{-2}$. The posterior probability distributions are shown in Extended Data Figure 5 and the parameter estimates are listed in Extended Data Table 7.

Bayesian model selection. In addition to an afterglow-only model, we consider an afterglow plus a KN and an afterglow plus a SN.

Kilonova. We use SEDs simulated by the multi-dimensional Monte Carlo radiative transfer code POSSIS ${ }^{185}$. The simulations are performed over a grid of KN parameters: dynamical ejecta $M_{\mathrm{ej}}^{\mathrm{dyn}}$, disk wind ejecta $M_{\mathrm{ej}}^{\text {wind }}$, opening angle $\Phi$, and the observation angle $\Theta_{\text {obs }}$ (see Ref. 20 for details). We use Gaussian process regression ${ }^{186,187}$ to interpolate the model, enabling rapid parameter inference.

Supernova. The SN model starts with a bicubic spline in time and frequency, $L_{\nu}^{\mathrm{SN}}(t, \nu)$, that interpolates a K-corrected SN1998bw template ${ }^{\sqrt{22}}$. We apply to the template a scale factor, $k$, and a stretch factor, $s$, which are drawn from a bivariate normal distribution that is consistent with a historical sample of GRB-SNe ${ }^{23}{ }^{190}$. The model for the observed flux density is

$$
F_{\nu}^{\mathrm{SN}}\left(t_{\mathrm{obs}}, \nu_{\mathrm{obs}}\right)=\frac{(1+z) k}{4 \pi d_{\mathrm{L}}{ }^{2}} L_{\nu}^{\mathrm{SN}}\left(\frac{t_{\mathrm{obs}}}{(1+z) s},(1+z) \nu_{\mathrm{obs}}\right)
$$

We perform Bayesian model selection to determine which model best explains the data. We used the nested sampling pipeline to calculate the Bayesian evidence for each of the three models. The Bayes factor, or the ratio of the evidences, between the afterglow-plus-KN model and the 
afterglow-only model is $\sim 1$, indicating that neither model is strongly favored over the other, because the $\mathrm{KN}$ contributes negligible flux compared to the afterglow at the time of the GMOS observation. The Bayes factor between the afterglow-plus-SN model and the afterglow-only model is $\sim 10^{5.5}$, strongly favoring the presence of a SN.

To better understand the source of discriminating power between models, we carry out a posterior predictive check. Here, we sample the posterior while excluding the GMOS $i$-band data point and then predict its value using the rest of the data. The posterior predictive distribution of the AB magnitude for the GMOS $i$-band detection both with and without the inclusion of a $\mathrm{SN}$ contribution is shown in Extended Data Figure 6. The fit with the $\mathrm{SN}$ is consistent with the observation, while the fit without the $\mathrm{SN}$ is inconsistent at the $\sim 5 \sigma$ level. Therefore, inclusion of the GMOS $i$-band data point requires a SN component, confirming a collapsar origin.

We compared our GMOS-N detection against extinction-corrected $i$-band fluxes of three GRB-KN candidates found in the literature ${ }^{191}$; using photometry from GRB $130603^{192}$ and GRB $160821^{193,194}$, and the compiled light curve of AT2017gfo ${ }^{5}$, we correct to the redshift of GRB 200826A. We fit each light curve to the best-fit 2D model of GW17081720 from POSS IS using SNCosmo ${ }^{197}$ and extract the corresponding $i$-band magnitude at a rest-frame time of 16 days. At $z=0.748$, both AT2017gfo and GRB 160821 would be at $\mathrm{M} \approx-10 \mathrm{mag}, \sim 8$ mags fainter than our detection. GRB 130603B does not have enough late-time detections at the same phase for comparison.

Our detection of a source with an extinction-corrected absolute magnitude in the $i$-band with $M_{i}=-18.0$ is consistent with the population of collapsars associated with LGRBs. A typical SN Ic reaches its peak magnitude 10 to 20 days post-burst, at $M_{B}=-17.66 \pm 1.18$ mag ${ }^{198}$. Fig. 3 shows K-corrected light curves for three well-sampled GRB-SNe: SN1998bw, one of the brightest; SN2006aj and SN2010bh, among the faintest. 

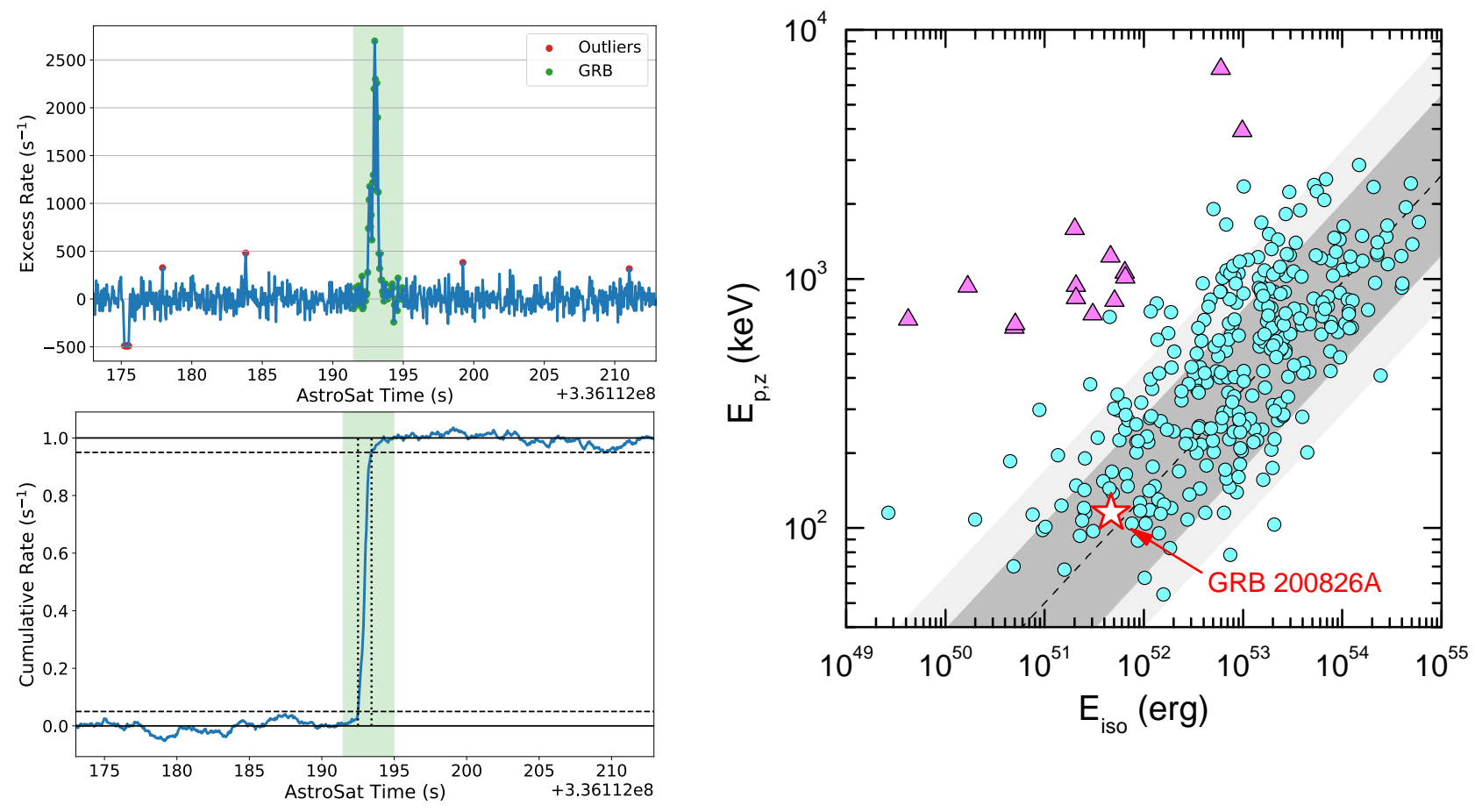

Extended Data Figure 1: The AstroSat and Konus-Wind gamma-ray detections. (left) Upper pannel panel: The de-trended light curve for GRB 200826A obtained from AstroSat CZTI data. We combined data from all four CZTI quadrants and binned it in $0.05 \mathrm{~s}$ bins. We fit and subtract a quadratic trend from the background to obtain zero-mean data. The shaded green region and corresponding green symbols denote a conservative GRB time span excluded from background trend estimation. Similarly red points denote outliers that are automatically flagged and rejected from the background estimate. Lower panel: A cumulative light curve obtained by summing the de-trended data, and normalised such that the median post-GRB value is 1.0. The dashed horizontal lines denote the 5\% and 95\% intensity levels. The corresponding vertical dotted black lines denote $T_{05}$ and $T_{95}$, yielding $T_{90}$ of $0.96_{-0.17}^{+0.71} \mathrm{~s}$. (right) Rest-frame energetics of 331 Konus-Wind GRBs (SGRB: triangles, LGRB: circles) with known redshift in the $E_{\text {iso }}-E_{\text {peak,z }}$ plane, with $E_{\text {peak }, \mathrm{z}}$ the rest frame $E_{\text {peak }}$. The hardness-intensity ('Amati') relation for LGRBs is plotted with its $68 \%$ and 90\% prediction intervals (dark and light gray regions, respectively). GRB 200826A, as a red star, appears not to be consistent with the SGRB population. 

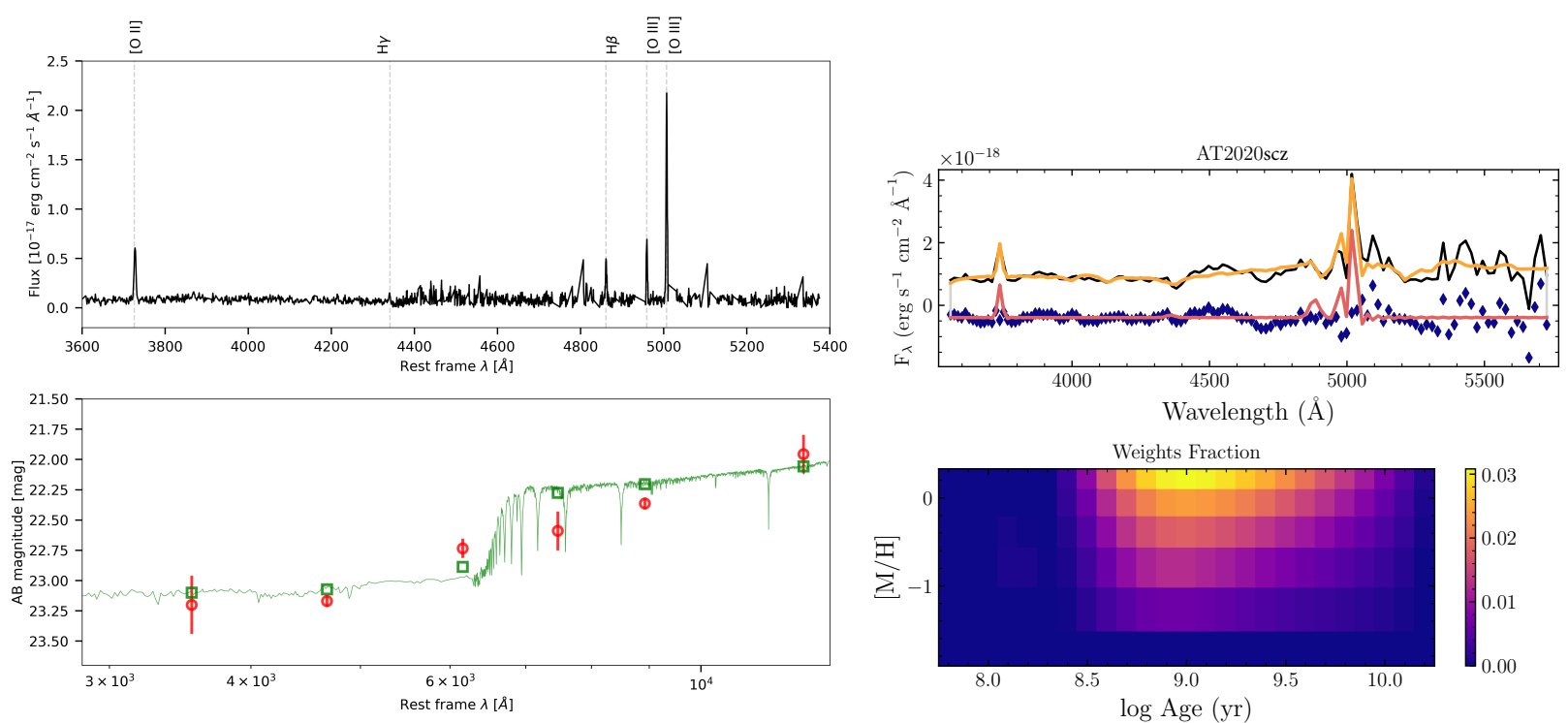

Extended Data Figure 2: The Host Galaxy. (left) In the upper panel, we show the GTC spectrum of the host galaxy and the lines used to determine a redshift of 0.748 . In the bottom panel, the photometry of the host galaxy (ugrizJ, see Extended Data Table 2) in the AB system is presented in red circles. The SED model and photometry from Prospector are shown in green. (right) The pPXF host galaxy model results described in §3. (top) The integrated spectrum (black) overlaid with the best-fit spectrum (orange), which sums the contributions of stars and gas in the modeled galaxy. The red spectrum shows the gas contribution to the spectrum, and the blue diamonds show the residuals to the fit. The gas is offset by $1.59 \mathrm{e}-18 \mathrm{erg} \mathrm{s}^{-1} \mathrm{~cm}^{-2}$. (bottom) The pPXF weights (color bar) of the different stellar population templates used to construct the best-fit galaxy. 


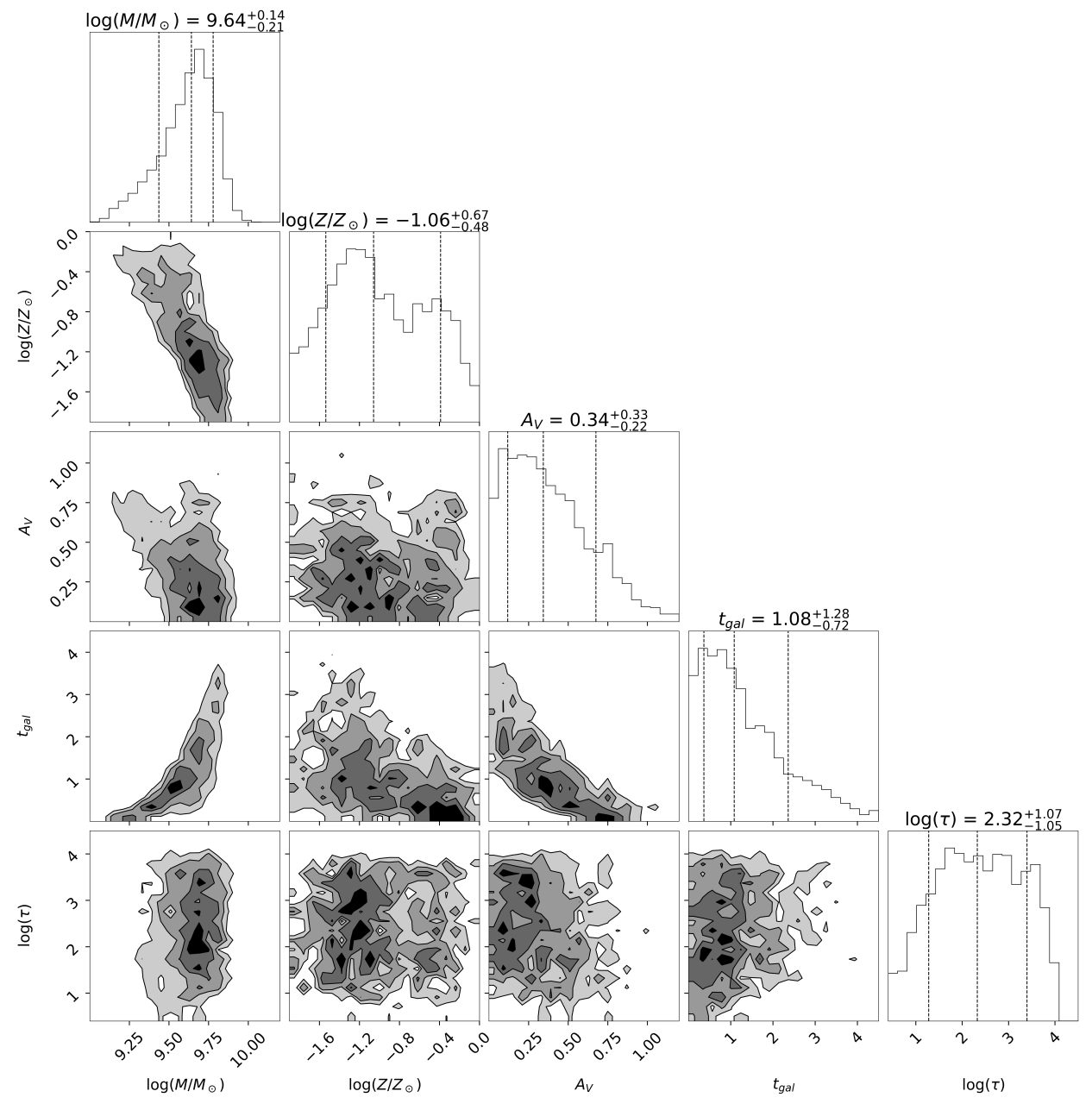

Extended Data Figure 3: Posterior distribution of the Prospector parameters. The covariances and posterior probability distributions of the parameters for the host galaxy derived form the Prospector modelling described in $\$ 3$. 


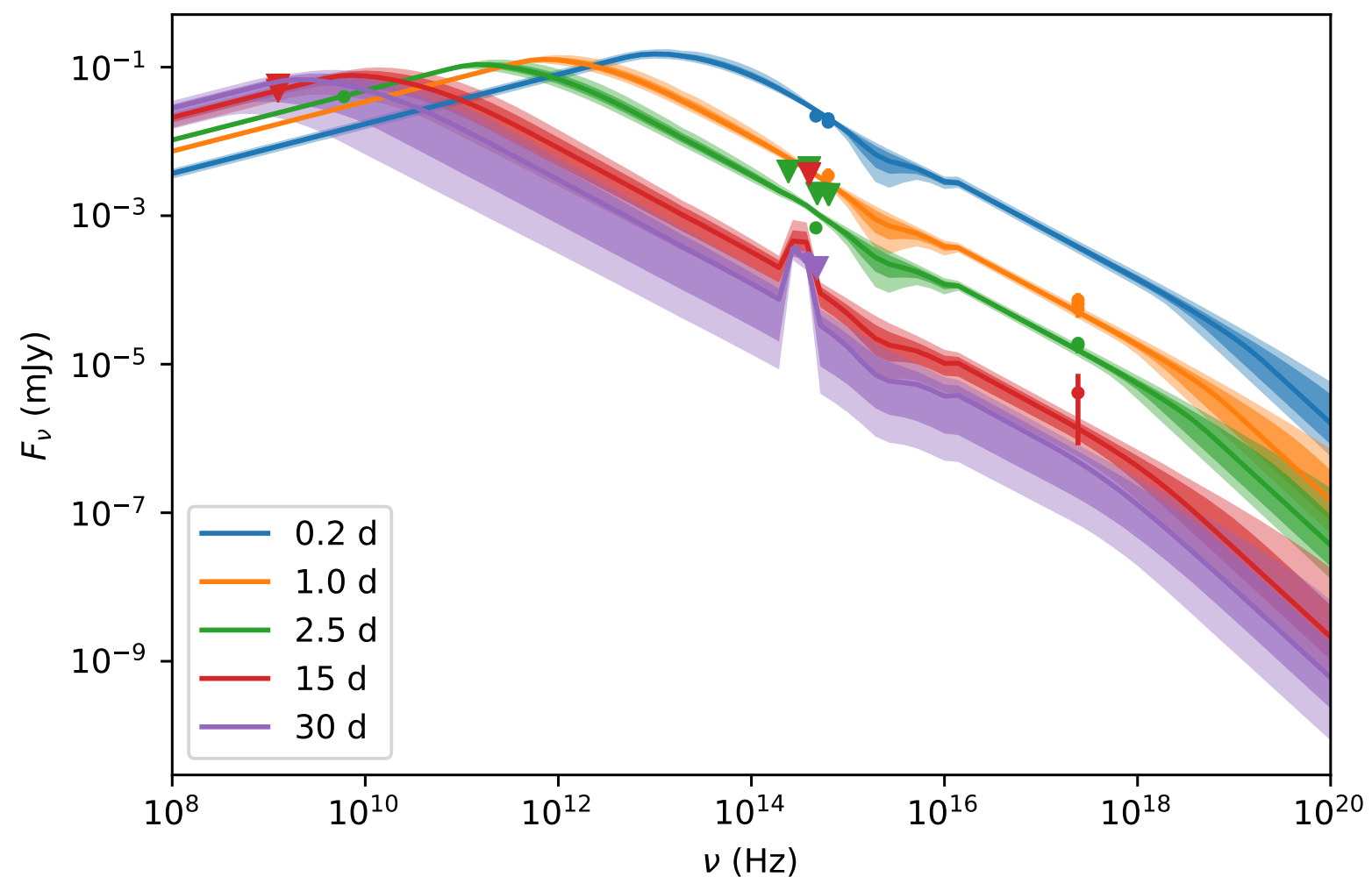

Extended Data Figure 4: SED sequence of the afterglow. The SED of our model compared to observations at five epochs. The cooling frequency $\nu_{c}$ is located at frequencies higher than $1 \mathrm{keV}$ (i.e. $\nu_{c}>2.4 \times 10^{17} \mathrm{~Hz}$ ). The glitches at optical $\nu \sim 10^{16} \mathrm{~Hz}$ are the edge of validity of our dust extinction model. The SN makes a large contribution at late times. See the observations in Extended Data Table 1Extended Data Table 3, and Extended Data Table 4.

\section{Data Availability}

Upon request, the corresponding author will provide data required to reproduce the figures, including light curves and spectra for any objects.

\section{Code Availability}

Upon request, the corresponding author will provide code (primarily in python) used to produce the figures. 


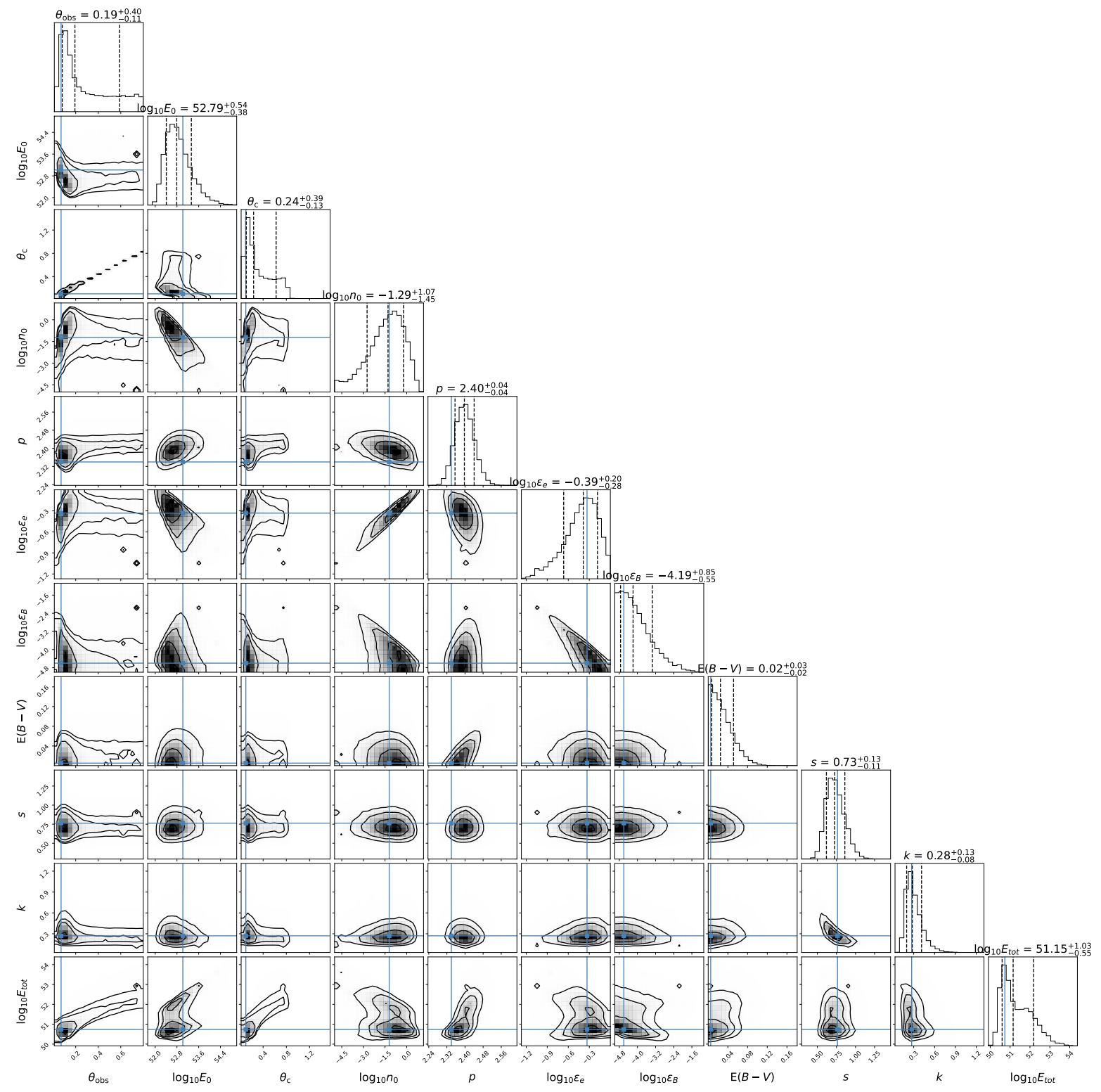

Extended Data Figure 5: Posterior distribution for the afterglowpy fit. The covariances and posterior probability distributions of the parameters for the top hat jet afterglow with 1998bw-like SN model described in $\$ 4$. The afterglow parameters are the viewing angle $\theta_{\text {obs }}(\mathrm{rad})$, on-axis isotropic kinetic energy $E_{0}(\mathrm{erg})$, opening angle $\theta_{c}(\mathrm{rad})$, circumburst number density $n_{0}\left(\mathrm{~cm}^{-3}\right)$, electron spectral index $p$, fraction of energy in accelerated electrons $\epsilon_{e}$, and fraction of energy in magnetic field $\epsilon_{B}$. We assume an SMC-like extinction curve with variable $E(B-V)$. The $\mathrm{SN}$ model is a $1998 \mathrm{bw}$ template with variable stretch $s$ and scale $k$. The total beaming-corrected kinetic energy in the jet $E_{\text {tot }}(\mathrm{erg})$, computed from the afterglow parameters, is also reported. The histograms denote the 14,50 , and 84 percentiles of the distributions, with blue lines marking the solution with maximum posterior probability density. 


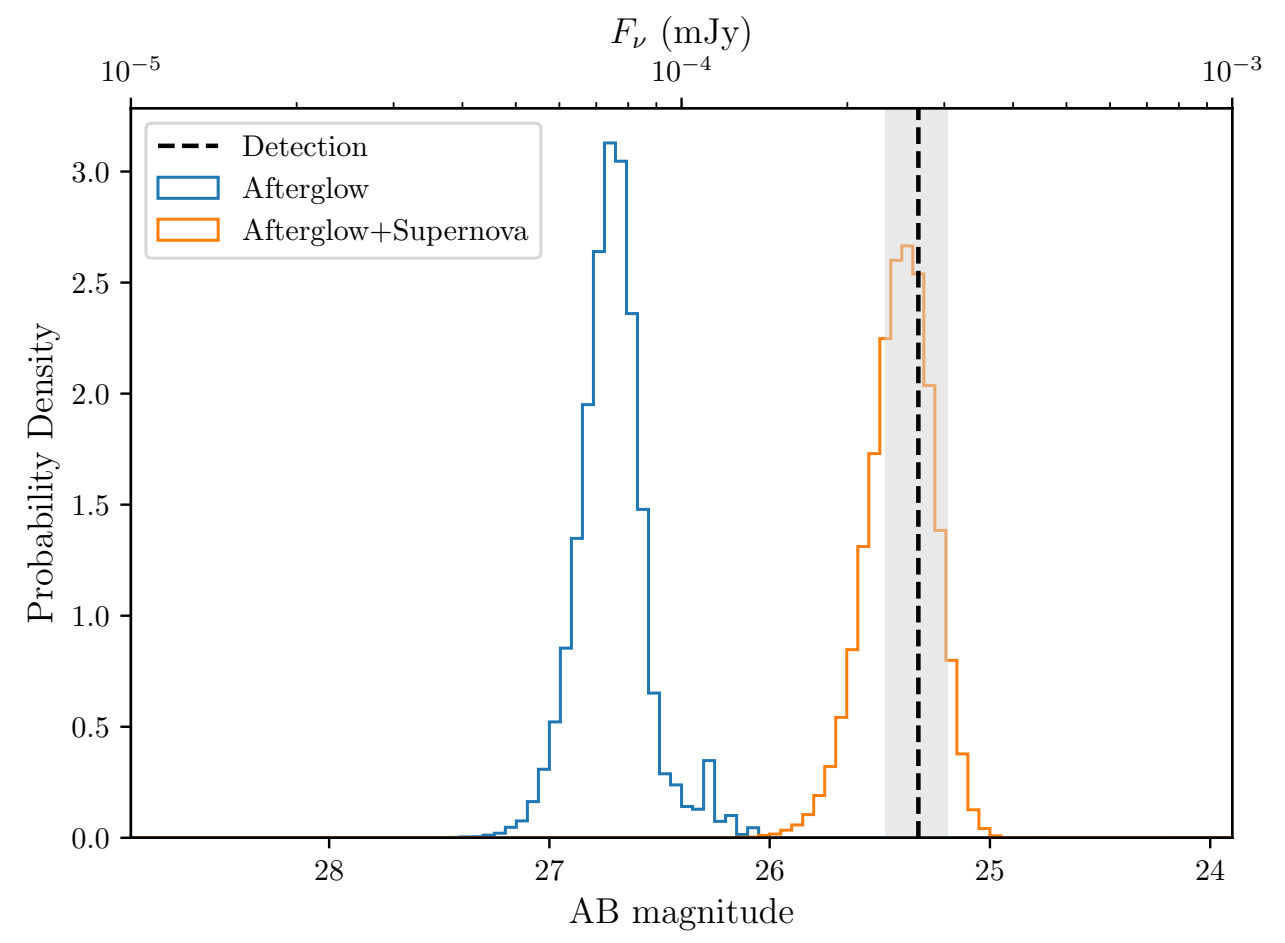

Extended Data Figure 6: Posterior predictive plot for the GMOS $i$-band detection. The posterior of the $\mathrm{AB}$ magnitude estimated at the time of the $i$-band data point $(\sim 28$ days after the trigger) using afterglow only (blue) and afterglow-and-SN (orange) light curves are shown. 


\begin{tabular}{cccccccc}
\hline Julian day & $\delta t$ & Instrument & Filter & AB magnitude & $\sigma_{m a g}$ & 5 $\sigma$ Limiting magnitude & $\mathbf{A}_{\nu}$ \\
\hline 2459085.8814 & -1.81 & P48+ZTF & g & - & - & 21.50 & 0.21 \\
2459085.9678 & -1.72 & P48+ZTF & r & - & - & 21.46 & 0.15 \\
2459086.9011 & -0.79 & P48+ZTF & r & - & - & 21.33 & 0.15 \\
2459086.9696 & -0.72 & P48+ZTF & g & - & - & 21.31 & 0.21 \\
2459087.8986 & 0.21 & P48+ZTF & g & 20.86 & 0.04 & 22.50 & 0.21 \\
2459087.9181 & 0.23 & P48+ZTF & r & 20.69 & 0.05 & 22.27 & 0.15 \\
2459087.9651 & 0.28 & P48+ZTF & g & 20.95 & 0.16 & 21.23 & 0.21 \\
2459088.8340 & 1.15 & P48+ZTF & g & 22.75 & 0.26 & 22.53 & 0.21 \\
2459088.9038 & 1.22 & P48+ZTF & r & - & - & 21.30 & 0.15 \\
2459088.9761 & 1.29 & P48+ZTF & g & - & - & 21.2 & 0.21 \\
2459089.6256 & 1.93 & P200+WIRC & J & - & - & 21.8 & 0.06 \\
2459089.9585 & 2.27 & FNT+LCO & r & - & - & 23.30 & 0.15 \\
2459089.9698 & 2.28 & FNT+LCO & g & - & - & 23.41 & 0.21 \\
2459090.9200 & 3.23 & LDT+LMI & r & 24.46 & 0.12 & 26.37 & 0.15 \\
2459115.9675 & 28.28 & Gemini+GMOS & i & 25.45 & 0.15 & 25.9 & 0.11 \\
2459115.9675 & 28.28 & Gemini+GMOS & r & - & - & 25.4 & 0.15 \\
2459133.8039 & 46.11 & Gemini+GMOS & i & - & - & 25.5 & 0.11 \\
2459133.8039 & 46.11 & Gemini+GMOS & r & - & - & 25.7 & 0.15 \\
\hline
\end{tabular}

Extended Data Table 1: Afterglow panchromatic observations. Observations of the GRB 200826A afterglow and SN. GRB 200826A was triggered at Julian day 2459087.6874.

\begin{tabular}{ccccccc}
\hline Julian day & $\delta t$ & Instrument & Filter & Host AB magnitude & $\sigma_{m a g}$ & $\mathbf{A}_{\nu}$ \\
\hline 2459089.62569 & 9.73 & P200+WIRC & $\mathrm{J}$ & $21.11^{*}$ & 0.16 & 0.05 \\
2459105.80457 & 18.11 & LMI+LDT & $\mathrm{u}$ & 23.45 & 0.24 & 0.28 \\
2459105.80457 & 18.11 & LMI+LDT & $\mathrm{g}$ & 23.36 & 0.05 & 0.22 \\
2459105.80457 & 18.11 & LMI+LDT & $\mathrm{r}$ & 22.86 & 0.18 & 0.15 \\
2459105.80457 & 18.11 & LMI+LDT & $\mathrm{i}$ & 22.66 & 0.16 & 0.11 \\
2459105.80457 & 18.11 & LMI+LDT & $\mathrm{z}$ & 22.13 & 0.05 & 0.09 \\
\hline
\end{tabular}

Extended Data Table 2: Host galaxy panchromatic data. Observations of the host galaxy of GRB 200826A. *Magnitudes are in the Vega system. 


\begin{tabular}{ccccc}
\hline Julian day & $\delta t$ & Instrument & Energy range & Count rate $\left[\mathbf{s}^{-1}\right]$ \\
\hline 2459088.386616 & 0.70 & XRT & $0.3-10 \mathrm{keV}$ & $2.16_{-0.53}^{0.53} \times 10^{-2}$ \\
2459088.441940 & 0.75 & XRT & $0.3-10 \mathrm{keV}$ & $1.91_{-0.49}^{0.49} \times 10^{-2}$ \\
2459088.522328 & 0.83 & XRT & $0.3-10 \mathrm{keV}$ & $1.66_{-0.41}^{0.41} \times 10^{-2}$ \\
2459089.437860 & 1.75 & XRT & $0.3-10 \mathrm{keV}$ & $5.64_{-1.21}^{1.21} \times 10^{-3}$ \\
2459090.285972 & 2.60 & XRT & $0.3-10 \mathrm{keV}$ & $5.36_{-1.22}^{1.22} \times 10^{-3}$ \\
2459094.090850 & 6.40 & XRT & $0.3-10 \mathrm{keV}$ & $3.33_{-0.77}^{0.77} \times 10^{-3}$ \\
2459104.352156 & 16.66 & XRT & $0.3-10 \mathrm{keV}$ & $1.22_{-0.67}^{0.98} \times 10^{-3}$ \\
\hline
\end{tabular}

Extended Data Table 3: Afterglow X-ray detections. X-ray observations of GRB 200826A.

\begin{tabular}{ccccc}
\hline Julian day & $\delta t$ & Instrument & Frequency & Flux $\left[\mathbf{e r g ~ c m}^{-2} \mathbf{s}^{-1}\right.$ ] \\
\hline 2459089.967407 & 2.28 & VLA & $6 \mathrm{GHz}$ & $40 * *$ \\
2459102.153825 & 14.46 & GMRT & $1.256 \mathrm{GHz}$ & $<48.6$ \\
2459107.485017 & 19.79 & GMRT & $1.256 \mathrm{GHz}$ & $<57.4$ \\
\hline
\end{tabular}

Extended Data Table 4: Radio data. Radio observations of GRB 200826A. ** VLA data from Ref. $\cdot 12$.

\begin{tabular}{|c|c|c|c|c|c|c|c|c|c|c|}
\hline Time Bins (s) & Model & Amplitude & $\begin{array}{l}E_{\text {peak }} \\
{[\mathbf{k e V}]}\end{array}$ & $\alpha$ & $\beta$ & $\begin{array}{l}\text { Photon Flux } \\
{\left[\mathbf{p h ~ s}^{-1} \mathbf{c m}^{-2}\right]}\end{array}$ & $\begin{array}{c}\text { Photon Fluence } \\
{\left[\mathbf{p h ~ c m}^{-2}\right]}\end{array}$ & $\begin{array}{l}\text { Energy Flux } \\
{\left[\operatorname{erg~s}^{-1} \mathbf{c m}^{-2}\right]}\end{array}$ & $\begin{array}{c}\text { Energy Fluence } \\
{\left[\mathrm{erg} \mathrm{cm}^{-2}\right]}\end{array}$ & Fit Merit \\
\hline $0.000-0.180$ & Comp & $0.3 \pm 0.04$ & $114.8 \pm 6.0$ & $-0.6 \pm 0.1$ & - & $32.2 \pm 0.9$ & $5.8 \pm 0.2$ & $3.1 \pm 0.2 \times 10^{-6}$ & $0.6 \pm 0.02 \times 10^{-6}$ & 1.1 \\
\hline $0.180-0.318$ & Band & $4.2 \pm 2.3$ & $61.3 \pm 0.5$ & $0.5 \pm 0.3$ & $-2.7 \pm 0.1$ & $41.5 \pm 1.1$ & $11.5 \pm 0.2$ & $3.7 \pm 0.2 \times 10^{-6}$ & $1.2 \pm 0.2 \times 10^{-6}$ & 0.9 \\
\hline $0.318-0.414$ & Band & $0.6 \pm 0.1$ & $141.9 \pm 10.3$ & $-0.5 \pm 0.1$ & $-3.0 \pm 0.4$ & $61.5 \pm 1.5$ & $17.4 \pm 0.2$ & $7.8 \pm 0.4 \times 10^{-6}$ & $1.9 \pm 0.3 \times 10^{-6}$ & 0.9 \\
\hline $0.414-0.506$ & Band & $0.7 \pm 0.1$ & $145.4 \pm 13.5$ & $-0.3 \pm 0.1$ & $-2.3 \pm 0.2$ & $61.4 \pm 1.5$ & $23.0 \pm 0.1$ & $9.6 \pm 0.4 \times 10^{-6}$ & $2.8 \pm 0.4 \times 10^{-6}$ & 1.0 \\
\hline $0.506-0.607$ & Band & $1.2 \pm 0.4$ & $99.6 \pm 7.4$ & $-0.04 \pm 0.2$ & $-2.5 \pm 0.2$ & $56.4 \pm 1.4$ & $28.8 \pm 0.1$ & $6.9 \pm 0.3 \times 10^{-6}$ & $3.5 \pm 0.3 \times 10^{-6}$ & 0.9 \\
\hline $0.607-0.747$ & Band & $0.9 \pm 0.2$ & $95.3 \pm 5.3$ & $-0.2 \pm 0.1$ & $-3.3 \pm 0.4$ & $41.7 \pm 1.1$ & $34.6 \pm 0.2$ & $4.2 \pm 0.2 \times 10^{-6}$ & $4.1 \pm 0.3 \times 10^{-6}$ & 0.9 \\
\hline $0.747-1.152$ & Band & $0.6 \pm 0.5$ & $41.2 \pm 3.9$ & $-0.3 \pm 0.4$ & $-2.7 \pm 0.2$ & $15.0 \pm 0.5$ & $40.7 \pm 0.2$ & $0.9 \pm 0.1 \times 10^{-6}$ & $4.5 \pm 0.3 \times 10^{-6}$ & 1.1 \\
\hline
\end{tabular}

Extended Data Table 5: Parameters of the Fermi-GBM fit. Fitting parameter of the Fermi-GBM gamma-ray spectrum of GRB 200826A.

\begin{tabular}{cc}
\hline Line & $F_{\nu}$ \\
$\AA$ & $10^{-17} \mathrm{erg} \mathrm{s}^{-1} \mathrm{~cm}^{-2}$ \\
\hline$[\mathrm{OII}]_{3726}$ & $1.268 \pm 0.37$ \\
{$[\mathrm{OII}]_{3729}$} & $2.522 \pm 0.37$ \\
{$[\mathrm{OIII}]_{5007}$} & $8.21 \pm 3.5$ \\
\hline
\end{tabular}

Extended Data Table 6: Host galaxy emission line fluxes. Fluxes derived with pPXF for the lines detected in the GTC spectrum of the host galaxy. 


\begin{tabular}{ccc}
\hline Parameter & Unit & Value \\
\hline$\theta_{v}$ & {$[\mathrm{rad}]$} & $0.20_{-0.15}^{+0.53}$ \\
$E_{\mathrm{K} \text {,iso }}$ & {$[\mathrm{erg}]$} & $6.0_{-4.4}^{+51.3} \times 10^{52}$ \\
$\theta_{c}$ & {$[\mathrm{rad}]$} & $0.24_{-0.17}^{+0.53}$ \\
$n$ & {$\left[\mathrm{~cm}^{-3}\right]$} & $5.5_{-5.4}^{+187.3} \times 10^{-2}$ \\
$p$ & & $2.40_{-0.07}^{+0.07}$ \\
$\epsilon_{e}$ & & $0.42_{-0.28}^{+0.40}$ \\
$\epsilon_{B}$ & & $6.4_{-5.2}^{+197.4} \times 10^{-5}$ \\
$E(B-V)$ & & $2.5_{-2.3}^{+4.8} \times 10^{-2}$ \\
stretch $s$ & & $0.73_{-0.16}^{+0.23}$ \\
scale $k$ & & $0.28_{-0.12}^{+0.25}$ \\
$E_{\mathrm{k}}$ & {$[\mathrm{erg}]$} & $1.4_{-1.2}^{+54.8} \times 10^{51}$ \\
\hline
\end{tabular}

Extended Data Table 7: Afterglow properties. Posterior afterglowpy fit model parameters with an SMC extinction curve, a SN1998bw template and including the final Gemini+GMOS detection. Uncertainties are quoted at $90 \% . E_{\mathrm{k}}$ is the beamed corrected kinetic energy. See $\$ 4$ for more details.

\section{References}

31. Zhang \& Choi. An analysis of the durations of swift gamma-ray bursts. Astronomy and Astrophysics 484, 293-297 (2008). URL https : / / doi .org/10 .1051/0004-6361: 20079210 .

32. Bromberg, O., Nakar, E., Piran, T. \& Sari, R. Short versus long and collapsars versus non-collapsars: A quantitative classification of gamma-ray bursts. The Astrophysical Journal 764, 179 (2013). URL http: / / stacks.iop.org/0004-637X/764/i=2/a=179.

33. Antonelli, L. A. et al. Grb 090426: the farthest short gamma-ray burst? Astronomy \& Astrophysics 507, L45-L48 (2009).

34. Levesque, E. M. et al. Grb 090426: The environment of a rest-frame 0.35-s gamma-ray burst at a redshift of 2.609. Monthly Notices of the Royal Astronomical Society 401, 963-972 (2010). 
35. Yang, B. et al. A possible macronova in the late afterglow of the long-short burst grb 060614. Nature Communications 6, 1-5 (2015).

36. Fynbo, J. P. et al. No supernovae associated with two long-duration $\gamma$-ray bursts. Nature 444, 1047-1049 (2006).

37. Gehrels, N. et al. The Swift Gamma-Ray Burst Mission. ApJ 611, 1005-1020 (2004). astro-ph/0405233.

38. Ricker, G. R. \& Vanderspek, R. K. Gamma-ray burst and afterglow astronomy 2001: A workshop celebrating the first year of the hete mission. AIPC 662 (2003).

39. Gehrels, N. et al. A short $\gamma$-ray burst apparently associated with an elliptical galaxy at redshift $\mathrm{z}=0.225$. Nature 437, 851-854 (2005). astro-ph/ 0505630 .

40. Hjorth, J. et al. The optical afterglow of the short $\gamma$-ray burst GRB 050709. Nature 437, 859-861 (2005). astro-ph/0510096.

41. Villasenor, J. et al. Discovery of the short $\gamma$-ray burst grb 050709. Nature 437, 855-858 (2005).

42. Berger, E. Short-Duration Gamma-Ray Bursts. ARA\&A 52, 43-105 (2014). 1311.2603.

43. Fong, W., Berger, E., Margutti, R. \& Zauderer, B. A. A decade of short-duration gamma-ray burst broadband afterglows: Energetics, circumburst densities, and jet opening angles. The Astrophysical Journal 815, 102 (2015). URL http: / / stacks . iop.org/ $0004-637 x / 815 / i=2 / a=102$.

44. Abbott, B. P. et al. GW170817: Observation of Gravitational Waves from a Binary Neutron Star Inspiral. Phys. Rev. Lett. 119, 161101 (2017). 1710.05832.

45. Meegan, C. et al. The fermi gamma-ray burst monitor. The Astrophysical Journal 702, 791-804 (2009). 0908.0450.

46. Abbott, B. P. et al. Gravitational Waves and Gamma-Rays from a Binary Neutron Star Merger: GW170817 and GRB 170817A. ApJ 848, L13 (2017). 1710.05834.

47. Goldstein, A. et al. An ordinary short gamma-ray burst with extraordinary implications: Fermi-gbm detection of grb 170817a. The Astrophysical Journal Letters 848, L14 (2017). 
48. Savchenko, V. et al. Integral detection of the first prompt gamma-ray signal coincident with the gravitational-wave event gw170817. The Astrophysical Journal Letters 848, L15 (2017).

49. Abbott, B. P. et al. Multi-messenger Observations of a Binary Neutron Star Merger. ApJ 848, L12 (2017). 1710.05833.

50. Kasliwal, M. M. et al. Illuminating gravitational waves: A concordant picture of photons from a neutron star merger. Science 358, 1559-1565 (2017). URL http:// science.sciencemag.org/content/358/6370/1559. http://science. sciencemag.org/content/358/6370/1559.full.pdf.

51. Arcavi, I. et al. Optical emission from a kilonova following a gravitational-wave-detected neutron-star merger. Nature 551, 64-66 (2017). 1710.05843.

52. Tanvir, N. R. et al. The Emergence of a Lanthanide-rich Kilonova Following the Merger of Two Neutron Stars. ApJ 848, L27 (2017). 1710 . 05455.

53. Chornock, R. et al. The Electromagnetic Counterpart of the Binary Neutron Star Merger LIGO/Virgo GW170817. IV. Detection of Near-infrared Signatures of r-process Nucleosynthesis with Gemini-South. ApJ 848, L19 (2017). 1710 . 05454.

54. Cowperthwaite, P. S. et al. The Electromagnetic Counterpart of the Binary Neutron Star Merger LIGO/Virgo GW170817. II. UV, Optical, and Near-infrared Light Curves and Comparison to Kilonova Models. ApJ 848, L17 (2017). 1710 . 05840.

55. Drout, M. R. et al. Light curves of the neutron star merger GW170817/SSS17a: Implications for r-process nucleosynthesis. Science 358, 1570-1574 (2017). 1710 . 05443.

56. Kasen, D., Metzger, B., Barnes, J., Quataert, E. \& Ramirez-Ruiz, E. Origin of the heavy elements in binary neutron-star mergers from a gravitational-wave event. Nature 551, 80-84 (2017). 1710.05463.

57. Pian, E. et al. Spectroscopic identification of r-process nucleosynthesis in a double neutron-star merger. Nature 551, 67-70 (2017). 1710.05858 .

58. Smartt, S. J. et al. A kilonova as the electromagnetic counterpart to a gravitational-wave source. Nature 551, 75-79 (2017). 1710.05841. 
59. Troja, E. et al. The X-ray counterpart to the gravitational-wave event GW170817. Nature 551, 71-74 (2017). 1710.05433.

60. Dichiara, S. et al. Short gamma-ray bursts within $200 \mathrm{mpc}$. Monthly Notices of the Royal Astronomical Society 492, 5011-5022 (2020).

61. Sakamoto, T. et al. The Second Swift Burst Alert Telescope Gamma-Ray Burst Catalog. ApJS 195, 2 (2011). 1104.4689.

62. von Kienlin, A. et al. The fourth fermi-gbm gamma-ray burst catalog: A decade of data. The Astrophysical Journal 893, 46 (2020). URL http://dx.doi.org/10.3847/ $1538-4357 / \mathrm{ab} 7 \mathrm{a} 18$.

63. Abbott, R. et al. GWTC-2: Compact Binary Coalescences Observed by LIGO and Virgo During the First Half of the Third Observing Run (2020). 2010.14527.

64. Nissanke, S., Sievers, J., Dalal, N. \& Holz, D. Localizing Compact Binary Inspirals on the Sky Using Ground-based Gravitational Wave Interferometers. ApJ 739, 99 (2011). 1105. 3184

65. Aasi, J. et al. Prospects for observing and localizing gravitational-wave transients with advanced ligo and advanced virgo. Living Reviews in Relativity 19 (2016).

66. Abbott, B. P. et al. Prospects for observing and localizing gravitational-wave transients with advanced ligo, advanced virgo and kagra. Living Reviews in Relativity 21, 3 (2018).

67. Law, N. M. et al. The Palomar Transient Factory: System Overview, Performance, and First Results. PASP 121, 1395 (2009). 0906.5350.

68. Singer, L. P. et al. Discovery and Redshift of an Optical Afterglow in $71 \mathrm{deg}^{2}$ : iPTF13bxl and GRB 130702A. ApJ 776, L34 (2013). 1307.5851.

69. Singer et al. The Needle in the $100 \mathrm{deg}^{2}$ Haystack: Uncovering Afterglows of Fermi GRBs with the Palomar Transient Factory. The Astrophysical Journal 806, 52 (2015). 1501. 00495 .

70. Bellm, E. C. et al. The zwicky transient facility: system overview, performance, and first results. Publications of the Astronomical Society of the Pacific 131, 018002 (2018). 
71. Graham, M. J. et al. The zwicky transient facility: science objectives. Publications of the Astronomical Society of the Pacific 131, 078001 (2019).

72. Masci, F. J. et al. The zwicky transient facility: Data processing, products, and archive. Publications of the Astronomical Society of the Pacific 131, 018003 (2018).

73. Coughlin, M. W. et al. 2900 square degree search for the optical counterpart of short gamma-ray burst grb 180523b with the zwicky transient facility. PASP 131, 048001 (2019).

74. Coughlin, M. W. et al. Growth on s190425z: searching thousands of square degrees to identify an optical or infrared counterpart to a binary neutron star merger with the zwicky transient facility and palomar gattini-ir. ApJ 885, L19 (2019).

75. Andreoni, I. et al. Growth on s190510g: Decam observation planning and follow-up of a distant binary neutron star merger candidate. ApJ 881, L16 (2019).

76. Kasliwal, M. M. et al. Kilonova luminosity function constraints based on zwicky transient facility searches for 13 neutron star mergers. arXiv preprint arXiv:2006.11306 (2020).

77. Anand, S. et al. Optical follow-up of the neutron star-black hole mergers S200105ae and S200115j. Nature Astronomy (2020). 2009.07210.

78. Cenko, S. B. et al. Discovery of a cosmological, relativistic outburst via its rapidly fading optical emission. ApJ 769, 130 (2013).

79. Cenko, S. B. et al. iptf14yb: the first discovery of a gamma-ray burst afterglow independent of a high-energy trigger. The Astrophysical Journal Letters 803, L24 (2015).

80. Ho, A. Y. et al. Ztf20aajnksq (at2020blt): A fast optical transient at $z \approx 2.9$ with no detected gamma-ray burst counterpart. arXiv:2006.10761 (2020).

81. Fermi GBM Team. GRB 200826A: Fermi GBM Final Real-time Localization. GRB Coordinates Network 28284, 1 (2020).

82. Pittori, C. et al. GRB 200826A: AGILE detection. GRB Coordinates Network 28289, 1 (2020).

83. Ridnaia, A. et al. Konus-Wind detection of GRB 200826A. GRB Coordinates Network 28294, 1 (2020). 
84. Gupta, S. et al. GRB 200826A: AstroSat CZTI detection. GRB Coordinates Network 28288, $1(2020)$.

85. Connaughton, V. et al. Localization of gamma-ray bursts using the fermi gamma-ray burst monitor. The Astrophysical Journal Supplement Series 216, 32 (2015). URL http: / / dx . doi.org/10.1088/0067-0049/216/2/32

86. Goldstein, A. et al. Evaluation of automated fermi gbm localizations of gamma-ray bursts. The Astrophysical Journal 895, 40 (2020). URL http://dx.doi.org/10.3847/ $1538-4357 / \mathrm{ab} 8 \mathrm{bdb}$.

87. Burgess, J. M., Yu, H.-F., Greiner, J. \& Mortlock, D. J. Awakening the BALROG (BAyesian Location Reconstruction Of GRBs): A new paradigm in spectral and location analysis of gamma ray bursts. arXiv e-prints arXiv:1610.07385 (2016). 1610.07385.

88. Band, D. et al. Batse observations of gamma-ray burst spectra. i-spectral diversity. The Astrophysical Journal 413, 281-292 (1993).

89. Gruber, D. et al. THE FERMI GBM GAMMA-RAY BURST SPECTRAL CATALOG: FOUR YEARS OF DATA. The Astrophysical Journal Supplement Series 211, 12 (2014).

90. Mangan, J., Dunwoody, R., Meegan, C. \& Fermi GBM Team. GRB 200826A: Fermi GBM observation. GRB Coordinates Network 28287, 1 (2020).

91. Yu, H.-F. et al. The fermi gbm gamma-ray burst time-resolved spectral catalog: brightest bursts in the first four years (2016). 1601.05206.

92. Guiriec, S. et al. TIME-RESOLVED SPECTROSCOPY OF THE THREE BRIGHTEST AND HARDEST SHORT GAMMA-RAY BURSTS OBSERVED WITH THEFERMIGAMMA-RAY BURST MONITOR. The Astrophysical Journal 725, 225-241 (2010). URL https: / / doi .org/10.10882F0004-637x2F 7252F12F225.

93. Kouveliotou, C. et al. Identification of Two Classes of Gamma-Ray Bursts. ApJ 413, L101 (1993).

94. Goldstein, A., Preece, R. D. \& Briggs, M. S. A new discriminator for gamma-ray burst classification: Theepeak-fluence energy ratio. The Astrophysical Journal 721, 1329-1332 (2010). URL http://dx.doi.org/10.1088/0004-637x/721/2/1329. 
95. Burns et al. (in prep).

96. Svinkin, D. S. et al. Classification of gamma-ray bursts observed with Konus-Wind. In Journal of Physics Conference Series, vol. 1400 of Journal of Physics Conference Series, 022010 (2019).

97. Svinkin, D. S. et al. The Second Konus-Wind Catalog of Short Gamma-Ray Bursts. ApJS 224, 10 (2016). 1603.06832 .

98. Rothberg, B., Kuhn, O., Veillet, C. \& Allanson, S. GRB 200826A. GRB Coordinates Network 28319, 1 (2020).

99. Tsvetkova, A. et al. The Konus-Wind Catalog of Gamma-Ray Bursts with Known Redshifts. I. Bursts Detected in the Triggered Mode. ApJ 850, 161 (2017). 1710.08746.

100. Amati, L. et al. Intrinsic spectra and energetics of bepposax gamma-ray bursts with known redshifts. Astronomy \& Astrophysics 390, 81-89 (2002).

101. Yonetoku, D. et al. Gamma-ray burst formation rate inferred from the spectral peak energy-peak luminosity relation. The Astrophysical Journal 609, 935 (2004).

102. Bhalerao, V. et al. The Cadmium Zinc Telluride Imager on AstroSat. Journal of Astrophysics and Astronomy 38, 31 (2017). 1608.03408.

103. Sharma, Y. et al. The search for fast transients with czti. arXiv preprint arXiv:2011.07067 (2020).

104. Coughlin, M. W. et al. Optimizing searches for electromagnetic counterparts of gravitational wave triggers. MNRAS 478, 692-702 (2018). 1803.02255.

105. Coughlin, M. W. et al. Optimizing Multi-Telescope Observations of Gravitational-Wave Counterparts. Mon. Not. R. Astron. Soc. (2019). URL https://doi.org/10. 1093/mnras/stz2485. Stz2485, http://oup.prod.sis.lan/mnras/ advance-article-pdf/doi/10.1093/mnras/stz2485/29808472/ stz2485.pdf.

106. Almualla, M. et al. Dynamic scheduling: target of opportunity observations of gravitational wave events. Mon. Not. R. Astron. Soc. 495, 4366-4371 (2020). URL 
https://doi.org/10.1093/mnras/staa1498, https://academic.oup. com/mnras/article-pdf/495/4/4366/33371783/staa1498.pdf,

107. Rana, J., Singhal, A., Gadre, B., Bhalerao, V. \& Bose, S. An enhanced method for scheduling observations of large sky error regions for finding optical counterparts to transients. The Astrophysical Journal 838, 108 (2017).

108. Burrows, D. N. et al. The Swift X-Ray Telescope. Space Sci. Rev. 120, 165-195 (2005). astro-ph/0508071.

109. Hurley, K. \& et al. IPN triangulation of GRB 200826A (short/bright) . GCN 28291 (2020).

110. Patterson, M. T. et al. The Zwicky Transient Facility Alert Distribution System. PASP 131, 018001 (2019). 1902.02227.

111. Kasliwal, M. et al. The growth marshal: a dynamic science portal for time-domain astronomy. Publications of the Astronomical Society of the Pacific 131, 038003 (2019).

112. Duev, D. A. et al. Real-bogus classification for the zwicky transient facility using deep learning. Monthly Notices of the Royal Astronomical Society 489, 3582-3590 (2019).

113. Nordin, J. et al. Transient processing and analysis using ampel: alert management, photometry, and evaluation of light curves. Astronomy \& Astrophysics 631, A147 (2019).

114. Soumagnac, M. T. \& Ofek, E. O. catsHTM: A Tool for Fast Accessing and Cross-matching Large Astronomical Catalogs. PASP 130, 075002 (2018). 1805.02666.

115. Stein, R. et al. A high-energy neutrino coincident with a tidal disruption event. arXiv e-prints arXiv:2005.05340 (2020). 2005.05340 .

116. Stein, R. \& Reusch, S. robertdstein/ampel_followup_pipeline: V1.1 release (2020). URL https://doi.org/10.5281/zenodo.4048336.

117. Chambers, K. C. et al. The Pan-STARRS1 Surveys. arXiv e-prints arXiv:1612.05560 (2016). 1612.05560 .

118. Tachibana, Y. \& Miller, A. A. A morphological classification model to identify unresolved panstarrs1 sources: Application in the ztf real-time pipeline. Publications of the Astronomical Society of the Pacific 130, 128001 (2018). 
119. Mahabal, A. et al. Machine learning for the zwicky transient facility. Publications of the Astronomical Society of the Pacific 131, 038002 (2019).

120. Cutri, R. M. et al. Explanatory Supplement to the AllWISE Data Release Products. Explanatory Supplement to the AllWISE Data Release Products (2013).

121. Wright, E. L. et al. The wide-field infrared survey explorer (wise): Mission description and initial on-orbit performance. The Astronomical Journal 140, 1868 (2010). URL http: //stacks.iop.org/1538-3881/140/i=6/a=1868.

122. Stern, D. et al. Mid-infrared selection of active galactic nuclei with the wide-field infrared survey explorer. i. characterizing wise-selected active galactic nuclei in cosmos. The Astrophysical Journal 753, 30 (2012). URL http: / / stacks.iop.org/0004-637X/ $753 / i=1 / a=30$.

123. Assef, R. J. et al. The WISE AGN Catalog. ApJS 234, 23 (2018). 1706.09901.

124. Yao, Y. et al. Ztf early observations of type ia supernovae. i. properties of the 2018 sample. The Astrophysical Journal 886, 152 (2019).

125. Jedicke, R., Bolin, B., Granvik, M. \& Beshore, E. A fast method for quantifying observational selection effects in asteroid surveys. Icarus 266, 173-188 (2016).

126. Sagues Carracedo, A. et al. GRB200826A: Zwicky Transient Facility Follow-Up of a Fermi Short GRB (Trigger 620108997). GRB Coordinates Network 28293, 1 (2020).

127. Ahumada, T. et al. GRB200826A: Zwicky Transient Facility Identifies Optical Afterglow Candidate of a Fermi Short GRB (Trigger 620108997). GRB Coordinates Network 28295, 1 (2020).

128. Stalder, B. et al. Observations of the grb afterglow atlas 17 aeu and its possible association with gw 170104. The Astrophysical Journal 850, 149 (2017).

129. Lipunov, V. et al. The master mobile astronomical system. optical observations of gamma-ray bursts. Astrophysics 48, 389-399 (2005).

130. Lipunov, V. et al. Fermi grb 200826a: Global master-net observations report. GRB Coordinates Network 28285, 1 (2020). 
131. Belkin, S. et al. GRB 200826A: Kitab optical observations. GRB Coordinates Network 28306, 1 (2020).

132. Bertin, E. \& Arnouts, S. SExtractor: Software for source extraction. A\&AS 117, 393-404 (1996).

133. Becker, A. HOTPANTS: High Order Transform of PSF ANd Template Subtraction (2015). 1504.004 .

134. Bertin, E. Automatic Astrometric and Photometric Calibration with SCAMP. In Gabriel, C., Arviset, C., Ponz, D. \& Enrique, S. (eds.) Astronomical Data Analysis Software and Systems XV, vol. 351 of Astronomical Society of the Pacific Conference Series, 112 (2006).

135. Wilson, J. C. et al. A Wide-Field Infrared Camera for the Palomar 200-inch Telescope. In Iye, M. \& Moorwood, A. F. M. (eds.) Instrument Design and Performance for Optical/Infrared Ground-based Telescopes, vol. 4841 of Society of Photo-Optical Instrumentation Engineers (SPIE) Conference Series, 451-458 (2003).

136. De, K. et al. Palomar Gattini-IR: Survey Overview, Data Processing System, On-sky Performance and First Results. PASP 132, 025001 (2020). 1910.13319.

137. Bertin, E. et al. The TERAPIX Pipeline. In Bohlender, D. A., Durand, D. \& Handley, T. H. (eds.) Astronomical Data Analysis Software and Systems XI, vol. 281 of Astronomical Society of the Pacific Conference Series, 228 (2002).

138. De, K. et al. The Zwicky Transient Facility Census of the Local Universe I: Systematic search for Calcium rich gap transients reveal three related spectroscopic sub-classes. arXiv e-prints arXiv:2004.09029 (2020). 2004.09029.

139. Massey, P. et al. As big and as good as it gets: The large monolithic imager for lowell observatory's 4.3-m discovery channel telescope. AAS 221, 345-02 (2013).

140. Ahumada, R. et al. The 16th Data Release of the Sloan Digital Sky Surveys: First Release from the APOGEE-2 Southern Survey and Full Release of eBOSS Spectra. ApJS 249, 3 (2020). 1912.02905 .

141. Labrie, K., Anderson, K., Cárdenes, R., Simpson, C. \& Turner, J. E. H. DRAGONS Data Reduction for Astronomy from Gemini Observatory North and South. In Teuben, P. J., 
Pound, M. W., Thomas, B. A. \& Warner, E. M. (eds.) Astronomical Data Analysis Software and Systems XXVII, vol. 523 of Astronomical Society of the Pacific Conference Series, 321 (2019).

142. Zackay, B., Ofek, E. O. \& Gal-Yam, A. Proper image subtraction-optimal transient detection, photometry, and hypothesis testing. The Astrophysical Journal 830, 27 (2016). URL http://stacks.iop.org/0004-637x/830/i=1/a=27.

143. Bertin, E. Automated Morphometry with SExtractor and PSFEx. In Evans, I. N., Accomazzi, A., Mink, D. J. \& Rots, A. H. (eds.) Astronomical Data Analysis Software and Systems XX, vol. 442 of Astronomical Society of the Pacific Conference Series, 435 (2011).

144. Guevel, D. \& Hosseinzadeh, G. Dguevel/Pyzogy: Initial Release (2017).

145. Gaia Collaboration et al. Gaia Data Release 2. Summary of the contents and survey properties. A\&A 616, A1 (2018). 1804.09365.

146. Fremling, C. et al. PTF12os and iPTF13bvn. Two stripped-envelope supernovae from low-mass progenitors in NGC 5806. A\&A 593, A68 (2016). 1606.03074.

147. Gal-Yam, A., Maoz, D., Guhathakurta, P. \& Filippenko, A. V. Supernovae in Low-Redshift Galaxy Clusters: Observations by the Wise Observatory Optical Transient Search (WOOTS). ApJ 680, 550-567 (2008). 0711.0808.

148. Ahumada, T. et al. GRB 200826A: GMOS-N detected source magnitude (Correction). GRB Coordinates Network 29029, 1 (2020).

149. Evans, P. et al. An online repository of swift/xrt light curves of-ray bursts. Astronomy \& Astrophysics 469, 379-385 (2007).

150. Evans, P. et al. Methods and results of an automatic analysis of a complete sample of swift-xrt observations of grbs. Monthly Notices of the Royal Astronomical Society 397, 1177-1201 (2009).

151. Willingale, R., Starling, R., Beardmore, A. P., Tanvir, N. R. \& O’Brien, P. T. Calibration of x-ray absorption in our galaxy. Monthly Notices of the Royal Astronomical Society 431, 394-404 (2013). 
152. D'Ai, A. et al. GRB 200826A: Swift-XRT afterglow detection. GRB Coordinates Network 28300, 1 (2020).

153. Alexander, K. D., Fong, W., Paterson, K. \& Rastinejad, J. GRB 200826A: VLA Detection at 6 GHz. GRB Coordinates Network 28302, 1 (2020).

154. Gupta, Y. et al. The upgraded gmrt: opening new windows on the radio universe. Curr. Sci 113, 707-14 (2017).

155. McMullin, J. P., Waters, B., Schiebel, D., Young, W. \& Golap, K. Casa architecture and applications. In Astronomical data analysis software and systems XVI, vol. 376, 127 (2007).

156. Chandra, P., Purkayastha, S., Bhalerao, V., Kumar, H. \& Kasliwal, M. GRB 200826A: uGMRT radio upper limit at 1.25 GHz. GRB Coordinates Network 28410, 1 (2020).

157. Cappellari, M. Improving the full spectrum fitting method: accurate convolution with Gauss-Hermite functions. MNRAS 466, 798-811 (2017). 1607 . 08538.

158. Schlafly, E. F. \& Finkbeiner, D. P. Measuring reddening with sloan digital sky survey stellar spectra and recalibrating sfd. The Astrophysical Journal 737, 103 (2011).

159. Johnson, B. D., Leja, J. L., Conroy, C. \& Speagle, J. S. Prospector: Stellar population inference from spectra and SEDs (2019). 1905.025.

160. Conroy, C., Gunn, J. E. \& White, M. The Propagation of Uncertainties in Stellar Population Synthesis Modeling. I. The Relevance of Uncertain Aspects of Stellar Evolution and the Initial Mass Function to the Derived Physical Properties of Galaxies. ApJ 699, 486-506 (2009). 0809.4261.

161. Conroy, C. \& Gunn, J. E. The Propagation of Uncertainties in Stellar Population Synthesis Modeling. III. Model Calibration, Comparison, and Evaluation. ApJ 712, 833-857 (2010). 0911.3151 .

162. Hinshaw, G. et al. Nine-year Wilkinson Microwave Anisotropy Probe (WMAP) Observations: Cosmological Parameter Results. ApJS 208, 19 (2013). 1212.5226.

163. Chabrier, G. Galactic Stellar and Substellar Initial Mass Function. PASP 115, 763-795 (2003). astro-ph/0304382. 
164. Calzetti, D. et al. The Dust Content and Opacity of Actively Star-forming Galaxies. ApJ 533, 682-695 (2000). astro-ph/9911459.

165. Speagle, J. S. dynesty: a dynamic nested sampling package for estimating bayesian posteriors and evidences. Monthly Notices of the Royal Astronomical Society 493, 3132-3158 (2020).

166. Nugent, A. E. et al. The distant, galaxy cluster environment of the short grb 161104a at $z \sim 0.8$ and a comparison to the short grb host population. arXiv preprint arXiv:2007.10372 (2020).

167. Paterson, K. et al. Discovery of the optical afterglow and host galaxy of short grb $181123 \mathrm{~b}$ at $\mathrm{z}=1.754$ : Implications for delay time distributions. The Astrophysical Journal Letters $\mathbf{8 9 8}$, L32 (2020).

168. O'Connor, B. et al. A tale of two mergers: constraints on kilonova detection in two short grbs at $\mathrm{z} \sim 0.5$. arXiv preprint arXiv:2012.00026 (2020).

169. Sari, R., Piran, T. \& Narayan, R. Spectra and Light Curves of Gamma-Ray Burst Afterglows. ApJ 497, L17-L20 (1998). astro-ph/9712005.

170. Granot, J. \& Sari, R. The Shape of Spectral Breaks in Gamma-Ray Burst Afterglows. ApJ 568, 820-829 (2002). astro-ph/0108027.

171. Zhang, B. et al. Physical Processes Shaping Gamma-Ray Burst X-Ray Afterglow Light Curves: Theoretical Implications from the Swift X-Ray Telescope Observations. ApJ 642, 354-370 (2006). astro-ph/0508321.

172. Sironi, L., Keshet, U. \& Lemoine, M. Relativistic Shocks: Particle Acceleration and Magnetization. Space Sci. Rev. 191, 519-544 (2015). 1506.02034.

173. Foreman-Mackey, D., Hogg, D. W., Lang, D. \& Goodman, J. emcee: The MCMC Hammer. PASP 125, 306 (2013). 1202.3665.

174. Buchner, J. et al. X-ray spectral modelling of the AGN obscuring region in the CDFS: Bayesian model selection and catalogue. A\&A 564, A125 (2014). 1402.0004.

175. Ryan, G., Eerten, H. v., Piro, L. \& Troja, E. Gamma-Ray Burst Afterglows in the Multimessenger Era: Numerical Models and Closure Relations. ApJ 896, 166 (2020). 1909.11691 . 
176. Troja, E. et al. The outflow structure of GW170817 from late-time broad-band observations. MNRAS 478, L18-L23 (2018). 1801.06516.

177. Cunningham, V. et al. Grb 160625b: Evidence for a gaussian-shaped jet. arXiv preprint arXiv:2009.00579 (2020).

178. Dietrich, T. et al. New constraints on the supranuclear equation of state and the hubble constant from nuclear physics - multi-messenger astronomy. Science 370, 1450-1453 (2020). 2002.11355.

179. Cash, W. Parameter estimation in astronomy through application of the likelihood ratio. ApJ 228, 939-947 (1979).

180. Pei, Y. C. Interstellar Dust from the Milky Way to the Magellanic Clouds. ApJ 395, 130 (1992).

181. Robitaille, T. P. et al. Astropy: A community Python package for astronomy. A\&A 558, A33 (2013).

182. Schady, P. Gamma-ray burst afterglows as probes of the ism. Journal of High Energy Astrophysics 7, 56-63 (2015).

183. Littlejohns, O. M. et al. A detailed study of the optical attenuation of gamma-ray bursts in the Swift era. MNRAS 449, 2919-2936 (2015). 1412.6530.

184. Zafar, T. et al. Vlt/x-shooter grbs: Individual extinction curves of star-forming regions. Monthly Notices of the Royal Astronomical Society 479, 1542-1554 (2018).

185. Bulla, M. POSSIS: predicting spectra, light curves, and polarization for multidimensional models of supernovae and kilonovae. MNRAS 489, 5037-5045 (2019). 1906.04205.

186. Coughlin, M. W. et al. Constraints on the neutron star equation of state from at $2017 \mathrm{gfo}$ using radiative transfer simulations. Mon. Not. Roy. Astron. Soc. 480, 3871-3878 (2018). 1805.09371 .

187. Coughlin, M. W. et al. Implications of the search for optical counterparts during the first six months of the Advanced LIGO's and Advanced Virgo's third observing run: possible limits on the ejecta mass and binary properties. Mon. Not. Roy. Astron. Soc. 492, 863-876 (2020). 1910.11246. 
188. Clocchiatti, A., Suntzeff, N. B., Covarrubias, R. \& Candia, P. The ultimate light curve of sn 1998bw/grb 980425. The Astronomical Journal 141, 163 (2011).

189. Cano, Z., Wang, S.-Q., Dai, Z.-G. \& Wu, X.-F. The observer's guide to the gamma-ray burst supernova connection. Advances in Astronomy 2017 (2017).

190. Klose, S. et al. Four grb supernovae at redshifts between 0.4 and 0.8 -the bursts grb $071112 \mathrm{c}$, 111228a, 120714b, and 130831a. AA 622, A138 (2019).

191. Kasliwal, M. M. et al. Illuminating gravitational waves: A concordant picture of photons from a neutron star merger. Science 358, 1559-1565 (2017). 1710.05436.

192. Berger, E., Fong, W. \& Chornock, R. An r-process kilonova associated with the short-hard grb 130603b. The Astrophysical Journal Letters 774, L23 (2013).

193. Troja, E. et al. The afterglow and kilonova of the short grb 160821b. Monthly Notices of the Royal Astronomical Society 489, 2104-2116 (2019).

194. Kasliwal, M. M., Korobkin, O., Lau, R. M., Wollaeger, R. \& Fryer, C. L. Infrared Emission from Kilonovae: The Case of the Nearby Short Hard Burst GRB 160821B. ApJ 843, L34 (2017). 1706.04647 .

195. Coulter, D. A. et al. Swope Supernova Survey 2017a (SSS17a), the optical counterpart to a gravitational wave source. Science 358, 1556-1558 (2017).

196. Villar, V. A. et al. The combined ultraviolet, optical, and near-infrared light curves of the kilonova associated with the binary neutron star merger gw170817: Unified data set, analytic models, and physical implications. The Astrophysical Journal Letters 851, L21 (2017). URL http://stacks.iop.org/2041-8205/851/i=1/a=L21.

197. Barbary, K. et al. SNCosmo: Python library for supernova cosmology (2016). 1611.017.

198. Richardson, D., Jenkins III, R. L., Wright, J. \& Maddox, L. Absolute-magnitude distributions of supernovae. The Astronomical Journal 147, 118 (2014). 